\title{
STEPANOV-LIKE PSEUDO ALMOST AUTOMORPHIC DYNAMICS OF QVRNNS WITH MIXED DELAYS ON TIME SCALES VIA A DIRECT METHOD
}

\author{
MOHSSINE ES-SAIYDY, MOHAMED ZITANE* \\ Moulay Ismaïl University, Faculty of Sciences, Department of Mathematics, \\ MACS laboratory, Meknès, Morocco \\ *Corresponding author: m.zitane@umi.ac.ma \\ Received Aug. 14, 2020
}

\begin{abstract}
In this paper, we are concerned with a class of quaternion-valued recurrent neutral neural networks (QVRNNs) with time-varying delays and infinite distributed delays on time scales. With the help of the Stepanov like pseudo almost automorphy on time scales, Banach's fixed point theorem, the theory of time scale calculations, and constructing a suitable Lyapunov functional, a set of sufficient criteria that guarantee the existence, uniqueness and the globally $S^{p}$-exponential stability of Stepanov-like pseudo almost automorphic solution on time scales of this class of neural networks are established via a direct method. In other words, we do not decompose the considered QVRNNs into real-valued systems or complex-valued systems. Finally, two numerical examples and simulations (for $\mathbb{T}=\mathbb{R}$ and $\mathbb{Z}$ ) are performed to verify our theoretical results.
\end{abstract}

2010 Mathematics Subject Classification. 43A60; 34C27; 34N05; 34C27; 34N05; 26A18; 26E70.

Key words and phrases. time scales; Bochner-like transform; quaternion-valued neural networks; Stepanov-like pseudo almost automorphy; global exponential stability.

\section{INTRODUCTION}

The artificial neural network has been used in recent decades to simulate the structure and function of the biological neural network. As a consequence, the neural networks (NNs) proposed by Chua and Yang ( [2]) have attracted growing attention because of their broad range of applications in, for example, function approximation, pattern recognition, associative memory, computing technology, nonlinear programming and combinatorial optimization ( $[22,24,27])$. Recently, recurrent neural networks (RNNs) have become a good tool for the approximating dynamical systems and are capable

DOI: 10.28924/APJM/7-32 
of learning the characteristics and modeling sequential data. They can be trained to reproduce any target dynamics, up to a certain degree of accuracy. As a result they have become of increasing interest to the various scientific research communities ( $[22,29,35])$. As is well known, the time delays that inevitably appear in both biological and artificial neural networks caused by the finite switching speed of neurons and amplifiers will affect the stability of neural networks. Moreover, the effect of time-delay on the dynamic behavior of systems has become a primary problem of delay, based on engineering technology and natural sciences ( $[13,15])$. Consequently, over the last decades, neural networks with different types of delay (discrete, distributed,...) and their dynamic characteristics have been widely studied ( [3,20,23]).

On the one hand, the theory of time scales, which originated in the work of Setefan Hilger in his Ph.D. thesis in 1988 ( [17]), has as its main goal the unification continuous analysis and discrete analysis. Therefore, the study of dynamic equations on time scales allows us to avoid producing separate results for differential equations on the one hand, and for difference equations on the other hand $([5,11,19,26])$. Hence, when we choose $\mathbb{T}=\mathbb{R}($ resp. $\mathbb{T}=\mathbb{Z})$, we get the result corresponding to the differential equations (resp. difference equations). Besides, since there are other time scales, not just the set of real numbers and the set of integers numbers, we have a much more general result. Furthermore, discrete recurrent neural networks are more practical for computation and numerical simulation than continuous recurrent neural networks. Then, it is useful to study the dynamics of neural networks on time scales( [10,32]).

On the other hand, almost automorphy is a very important and powerful dynamic behavior of neural networks that have been extensively investigated by a number of researchers $([8,9,34,36,37])$. The notion of Stepanov-like pseudo almost automorphy ( $\left.S^{p} P A A\right)$ on time scales, which is a natural generalization of the concepts of pseudo almost periodic, pseudo almost automorphic, Stepanov-like almost automorphic and Stepanov-like pseudo almost periodic and much more general and plays a very important role in better understanding the almost periodicity, was introduced in the literature by $\mathbf{M}$. Es-saiydy an M. Zitane ( [12]).

Moreover, in 1840, the Irish mathematician Hamilton ( [18]) invented the quaternion algebra, it includes real numbers and complex numbers. Quaternion algebra has been widely used in a variety of fields, including theoretical physics, modern mathematics, geometry, NNs, image processing and robotics ( $[16,21,25])$. Another practical application of applying quaternion is that it can exploit and treat 3-or 4-dimensional vectors in a single entity, which facilitates computation in 3-or 4-dimensional situations, so that the efficient processing of information can be carried out through quaternionic 
variable operations. Nevertheless, because the multiplication of quaternion numbers does not satisfy the commutative law, the research of quaternion-valued NNs (QVNNs) has had great difficulties. In addition, most of the existing results are obtained by decomposing QVNNs into real-VNNs ( [7,33]) or a complex-VNNs ( $[14,28])$. As a result, current results on QVNNs dynamics through direct methods are still very rare.

To the best of our knowledge, no such work has been done on the $S^{p} P A A$ solution and $S^{p}$-globally exponential stability of real-valued RNNs, complex-valued RNNs or quaternion-valued RNNs on time scales. It is therefore a difficult and important problem in theories and applications. Motivated by the above analysis and discussion, the main purpose of this paper is to study the existence and $S^{p}$-global exponential stability of $S^{p} P A A$ solution of quaternion-valued recurrent neural networks (QVRNNs) with mixed time-varying delays on time scales by direct methods. Further, our methods proposed in this paper can be used to study the problem of Stepanov-like almost periodic solution and Stepanov-like almost automorphic solution for other types of discrete- or continuous- QVNNs such as quaternion-valued Hopfield NNs, Cohen-Grossberg NNs and quaternion-valued BAM.

This paper is organized as follows: In section 2, the QVRNNs with mixed time-varying delays on time scales are presented and we introduce some necessary definitions and lemmas which are needed in later sections. In section 3, we establish some sufficient conditions for the existence and $S^{p}$-global exponential stability of $S^{p} P A A$ solution for QVRNNs with mixed time-varying delays on time scales. In section 4, two numerical examples and simulations are given to demonstrate the feasibility of our theoretical results.

\section{MODEL DESCRIPTION AND PRELIMINARIES}

In this section, we shall first recall some fundamental definitions, lemmas which are used in what follows. Throughout this paper we fix $p \geq 1$ and $(X,\|\|$.$) is a Banach space. We denote by \mathbb{N}, \mathbb{Z}, \mathbb{R}$, $\mathbb{C}$ and $\mathbb{H}$ the set of positive integers, the set of integers, the set of real, the set of complex numbers and the algebra of quaternion respectively. The skew field of the quaternion is determined by $\mathbb{H}:=$ $\left\{x ; x=x^{R}+x^{I} i+x^{J} j+x^{K} k\right\}$, where $x^{R}, x^{I}, x^{J}$ and $x^{K}$ are real numbers and the elements $i, j$, and $k$ obey the Hamilton's multiplication rules: $i j=-j i=k, j k=-k j=i, k i=-i k=j, i^{2}=j^{2}=k^{2}=-1$. Moreover, the quaternion conjugate is defined as $\bar{x}=x^{R}-x^{I} i-x^{J} j-x^{K} k$, and the norm of $x$ is defined by $|x|_{\mathbb{H}}=\sqrt{x \bar{x}}$. 
In this paper, we consider the following quaternion-valued recurrent neural networks (QVRNNs) with mixed time-varying delays on time scales:

$$
\begin{aligned}
x_{l}^{\Delta}(t) & =-a_{l}(t) x_{l}(t)+\sum_{m=1}^{n} b_{l m}(t) f_{m}\left(x_{m}(t)\right)+\sum_{m=1}^{n} c_{l m}(t) g_{m}\left(x_{m}\left(t-\xi_{m}(t)\right)\right) \\
& +\sum_{m=1}^{n} d_{l m}(t) \int_{-\infty}^{t} N_{l m}(t-z) h_{m}\left(x_{m}(z)\right) \Delta z+I_{l}(t), \quad t \in \mathbb{T} .
\end{aligned}
$$

Where $l \in\{1,2, \ldots, n\}, n$ corresponds to the number of units in neural networks, $\mathbb{T}$ is an almost periodic time scale; $\mathbb{H}$ is a Quaternion algebra; $x_{l}(t) \in \mathbb{H}$ corresponds to the state of the lth unit at time $t$, $a_{l}(t)=\operatorname{diag}\left\{a_{i}(t)\right\}_{i=1}^{i=n}$ represents the rate with which the ith neuron will reset its potential to the resting state in isolation when they are disconnected from the network and the external inputs at time $t, f_{m}$, $g_{m}$ and $h_{m}: \mathbb{H} \rightarrow \mathbb{H}$ are output transfer functions, $b_{l m}(),. c_{l m}(),. d_{l m}($.$) present the connection weights,$ the discretely delayed connection weights, and the distributively delayed connection weights, of the $m t h$ neuron on the $l$ neuron, respectively. $\xi_{m}($.$) corresponds to transmission delays at time t$ and satisfy $t-\xi_{m}(t) \in \mathbb{T}$ for $t \in \mathbb{T}, N_{l m}$ is the delay kernel function, $I_{l}($.$) denote the state bias of the l t h$ neuron. The initial condition of system (2.1) is of the form

$$
x_{l}(s)=\psi_{l}(s), \quad s \in(-\infty, 0]_{\mathbb{T}}
$$

where $\psi_{l}$ is rd-continuous and $\psi_{l} \in L_{l o c}^{p}\left((-\infty, 0]_{\mathbb{T}}, \mathbb{H}\right) l=1, \ldots, n$.

Remark 2.1. 1) If $\mathbb{T}=\mathbb{Z}$, Then sys.(2.1) can be transformed into the form below :

$$
\begin{aligned}
x_{l}(k+1)-x_{l}(k) & =-a_{l}(k) x_{l}(k)+\sum_{m=1}^{n} b_{l m}(k) f_{m}\left(x_{m}(k)\right) \\
& +\sum_{m=1}^{n} c_{l m}(k) g_{m}\left(x_{m}\left(k-\xi_{m}(k)\right)\right) \\
& +\sum_{m=1}^{n} d_{l m}(k) \int_{-\infty}^{k} N_{l m}(k-z) h_{m}\left(x_{m}(k)\right) d z+I_{l}(k), \quad k \in \mathbb{Z} .
\end{aligned}
$$

2) If $\mathbb{T}=\mathbb{R}$, Then sys.(2.1) can be transformed into the form below :

$$
\begin{aligned}
x_{l}^{\prime}(t) & =-a_{l}(t) x_{l}(t)+\sum_{m=1}^{n} b_{l m}(t) f_{m}\left(x_{m}(t)\right)+\sum_{m=1}^{n} c_{l m}(t) g_{m}\left(x_{m}\left(t-\xi_{m}(t)\right)\right) \\
& +\sum_{m=1}^{n} d_{l m}(t) \int_{-\infty}^{t} N_{l m}(t-z) h_{m}\left(x_{m}(z)\right) d z+I_{l}(t), \quad t \in \mathbb{R} .
\end{aligned}
$$

\subsection{Time scales.}

Definition 2.1 ( [6]). An arbitrary nonempty closed subset $\mathbb{T}$ of the set of real numbers $\mathbb{R}$ is called a time scale. The forward and backward jump operators $\sigma, \psi: \mathbb{T} \longrightarrow \mathbb{T}$ and the graininess $\mu: \mathbb{T} \longrightarrow \mathbb{R}^{+}$ 
are defined, respectively, by $\sigma(t)=\inf \{s \in \mathbb{T}: s>t\}, \psi(t)=\sup \{s \in \mathbb{T}: s<t\}, \mu(t)=\sigma(t)-t$. A point $t \in \mathbb{T}$ is called left-dense if $t>\inf \mathbb{T}$ and $\psi(t)=t$, left-scattered if $\psi(t)<t$, right-dense if $t<\sup \mathbb{T}$ and $\sigma(t)=t$, and right-scattered if $\sigma(t)>t$. A function $f: \mathbb{T} \longrightarrow \mathbb{R}$ is called right-dense continuous or rd-continuous provided that it is continuous at all right-dense points in $\mathbb{T}$ and its left-side limits exist (finite) at left-dense points in $\mathbb{T}$. A function $f: \mathbb{T} \longrightarrow \mathbb{R}$ is called continuous if and only if it is both left-dense continuous and right-dense continuous. A function $p: \mathbb{T} \longrightarrow \mathbb{R}$ is called regressive provided if $1+\mu(t) p(t) \neq 0$ for all $t \in \mathbb{T} \backslash \max (\mathbb{T})$. The set of all regressive and rd-continuous functions $p: \mathbb{T} \longrightarrow \mathbb{R}$ will be denoted by $\mathfrak{R}=\mathfrak{R}(\mathbb{T})=\mathfrak{R}(\mathbb{T} ; \mathbb{R})$. We define the set $\mathfrak{R}^{+}$of all positively regressive elements by $\mathfrak{R}^{+}=\mathfrak{R}^{+}(\mathbb{T})=\mathfrak{R}^{+}(\mathbb{T} ; \mathbb{R})=\{p \in \mathfrak{R}: 1+\mu(t) p(t)>0$ for all $t \in \mathbb{T}\}$. Let $a, b \in \mathbb{T}$, with $a \leq b,[a, b],[a, b),(a, b],(a, b)$ being the usual intervals on the real line. The intervals $[a, a),(a, a]$, $(a, a)$ are understood as the empty set, and we use the following symbols :

$$
\begin{aligned}
& {[a, b]_{\mathbb{T}}=[a, b] \cap \mathbb{T} \quad[a, b)_{\mathbb{T}}=[a, b) \cap \mathbb{T}} \\
& (a, b]_{\mathbb{T}}=(a, b] \cap \mathbb{T} \quad(a, b)_{\mathbb{T}}=(a, b) \cap \mathbb{T} .
\end{aligned}
$$

Definition 2.2 ( [6]). A time scale $\mathbb{T}$ is called invariant under translations if

$$
\Pi=\{\tau \in \mathbb{R}: t \pm \tau \in \mathbb{T} ; \forall t \in \mathbb{T}\} \neq\{0\}
$$

Definition 2.3 ( [6]). For $f: \mathbb{T} \rightarrow X$ and $s \in \mathbb{T} \backslash\{\max \mathbb{T}\}, f^{\Delta}(t) \in X$ is the delta derivative of $f$ at $s$ if for $\varepsilon>0$, there is a neighborhood $V$ of $s$ such that for $t \in V$,

$$
\left\|f(\sigma(s))-f(t)-f^{\Delta}(s)(\sigma(s)-t)\right\|<\varepsilon|\sigma(s)-t| .
$$

Moreover, $f$ is delta differentiable on $\mathbb{T}$ provided that $f^{\Delta}(s)$ exists for $s \in \mathbb{T}$.

Lemma 2.1 ( [6]). Considering that $f, g$ be delta differentiable functions on $\mathbb{T}$, then:

1) $\left(\lambda_{1} f+\lambda_{2} g\right)^{\Delta}=\lambda_{1} f^{\Delta}+\lambda_{2} g^{\Delta}$, for any constants $\lambda_{1}, \lambda_{2}$;

2)

$$
\begin{aligned}
(f g)^{\Delta}(t) & =(f)^{\Delta}(t) g(t)+f(\sigma(t))(g)^{\Delta}(t) \\
& =f(t)(g)^{\Delta}(t)+(f)^{\Delta}(t) g(\sigma(t))
\end{aligned}
$$

3) If $f$ and $f^{\Delta}$ are continuous, then

$$
\left(\int_{a}^{t} f(t, s) \Delta s\right)^{\Delta}=f(\sigma(t), t)+\int_{a}^{t} f(t, s) \Delta s .
$$


Definition 2.4 ( [6]). If $p \in \Re$, then we define the exponential function by :

$$
\hat{e}_{p}(t, s)=\exp \left\{\int_{s}^{t} \xi_{\mu(\tau)}(p(\tau)) \Delta \tau\right\},
$$

for $s, t \in \mathbb{T}$, with the cylinder transformation

$$
\xi_{m}(z)= \begin{cases}\frac{\log (1+h z)}{h}, & \text { if } h \neq 0, \\ z, & \text { if } h=0 .\end{cases}
$$

Definition 2.5 ( [6]). If $p, q \in \mathfrak{R}$, then we define a circle plus addition by

$$
(p \oplus q)(t):=p(t)+q(t)+p(t) q(t) \mu(t)
$$

for all $t \in \mathbb{T} \backslash \max (\mathbb{T})$. For $p \in \mathfrak{R}$, define a circle minus $p$ by

$$
\ominus p=-\frac{p}{1+\mu p}
$$

Lemma 2.2 ( [6]). Let $p, q \in \Re$, and $t, s, r \in \mathbb{T}$. Then,

1) $\hat{e}_{0}(t, s)=1$ and $\hat{e}_{p}(t, t)=1$;

2) $\hat{e}_{p}(\sigma(t), s)=(1+p(t) \mu(t)) \hat{e}_{p}(t, s)$;

3) $\hat{e}_{p}(t, s)=\frac{1}{\hat{e}_{p}(s, t)}=\hat{e}_{\ominus p}(s, t)$;

4) $\hat{e}_{p}(t, r) \hat{e}_{p}(r, s)=\hat{e}_{p}(t, s)$;

5) $\left(\hat{e}_{p}(t, s)\right)^{\Delta}=p(t) \hat{e}_{p}(t, s)$;

6) If $a, b, c \in \mathbb{T}$. Then,

$$
\int_{a}^{b} \hat{e}_{p}(c, \sigma(t)) p(t) \Delta t=\hat{e}_{p}(c, a)-\hat{e}_{p}(c, b) .
$$

7) For $t_{0} \in \mathbb{T}, \hat{e}_{\ominus \lambda}\left(t_{0},.\right)$ is increasing on $\left(-\infty, t_{0}\right]_{\mathbb{T}}$.

Lemma 2.3 ( [6]). Assume $p \in \mathfrak{R}$, and $t_{0} \in \mathbb{T}$. If $1+\mu(t) p(t)>0$ for all $t \in \mathbb{T}$, then, $\hat{e}_{p}\left(t, t_{0}\right)>0$ for all $t \in \mathbb{T}$.

Definition 2.6 ([1]). Let $F_{1}=\left\{\left[t, s[\mathbb{T}: t, s \in \mathbb{T}\right.\right.$ with $\quad t \leq s\}$. Define a countably additive measure $m_{1}$ on $F_{1}$ by assigning to every $\left[t, s\left[\mathbb{T} \in F_{1}\right.\right.$ its length, i.e;

$$
m_{1}([t, s[\mathbb{T})=s-t
$$

Using $m_{1}$, we can generate the outer measure $m_{1}^{*}$ on $P(\mathbb{T})$ ( the power set of $\mathbb{T}$ ): for $E \in P(\mathbb{T})$,

$$
m_{1}^{*}(E)= \begin{cases}\inf _{\mathfrak{B}}\left\{\sum_{i \in I_{B}}\left(s_{m}-t_{m}\right)\right\} \in \mathbb{R}^{+}, & \beta \notin E, \\ +\infty, & \beta \in E,\end{cases}
$$


where $\beta=\sup \mathbb{T}$, and,

$$
\mathfrak{B}=\left\{\left\{\left[t_{m}, s_{m}\left[\mathbb{T} \in F_{1}\right\}_{i \in I_{B}}: I_{B} \subset \mathbb{N}, E \subset \cup_{i \in I_{B}}\left[t_{m}, s_{m}[\mathbb{T}\}\right.\right.\right.\right.
$$

A set $A \subset \mathbb{T}$ is called $\Delta$-measurable if for $E \subset \mathbb{T}$,

$$
m_{1}^{*}(E)=m_{1}^{*}(E \cap A)+m_{1}^{*}(E \cap(\mathbb{T} \backslash A)) .
$$

Let

$$
\mathscr{M}^{*}\left(m_{1}^{*}\right)=\{A, A \text { is } \Delta-\text { measurable subset in } \mathbb{T}\}
$$

Restricting $m_{1}^{*}$ to $\mathscr{M}^{*}\left(m_{1}^{*}\right)$, we get the Lebesgue $\Delta$-measure, which is denoted by $\mu_{\Delta}$.

Definition 2.7 ( [1]). $f: \mathbb{T} \rightarrow X$ is a $\Delta$-measurable function if there exists a simple function sequence $\left\{f_{k}: k \in \mathbb{N}\right\}$ such that, $f_{k}(s) \rightarrow f(s)$ a.e. in $\mathbb{T}$.

Definition 2.8 ( [1]). $f: \mathbb{T} \rightarrow X$ is a $\Delta$-integrable function if there exists a simple function sequence $\left\{f_{k}: k \in \mathbb{N}\right\}$ such that $f_{k}(s) \rightarrow f(s)$ a.e. in $\mathbb{T}$ and,

$$
\lim _{k \rightarrow \infty} \int_{\mathbb{T}}\left\|f_{k}(s)-f(s)\right\| \Delta s=0
$$

Then, the integral of $f$ is defined as

$$
\int_{\mathbb{T}} f(s) \Delta s=\lim _{k \rightarrow \infty} \int_{\mathbb{T}} f_{k}(s) \Delta s
$$

Definition 2.9 ( [1]). For $p \geq 1, f: \mathbb{T} \rightarrow X$ is called locally $L^{p} \Delta$-integrable if $f$ is $\Delta$-measurable and for any compact $\Delta$-measurable set $E \subset \mathbb{T}$, the $\Delta$-integral

$$
\int_{E}\|f(s)\|^{p} \Delta s<\infty
$$

The set of all $L^{p} \Delta$-integrable functions is denoted by $L_{l o c}^{p}(\mathbb{T} ; X)$.

Theorem 2.1 ( [4]). If $a, b \in \mathbb{T}$, with $a \leq b$, then,

1) $\mu_{\Delta}([a, b))=b-a$,

2) $\mu_{\Delta}((a, b))=b-\sigma(a)$.

Theorem 2.2 ( [4]). If $a, b \in \mathbb{T} \backslash\{\max \mathbb{T}\}$, with $a \leq b$, then,

1) $\mu_{\Delta}((a, b])=\sigma(b)-\sigma(a)$,

2) $\mu_{\Delta}([a, b])=\sigma(b)-a$. 
2.2. Stepanov-like pseudo almost automorphic functions on $\mathbb{T}$. This subsection is devoted to definitions, the important properties of Stepanov-like pseudo almost automorphic functions on time scales introduced by M. Es-saiydy and M. Zitane ( [12]).

Definition 2.10 ([12]). We say that $f: \mathbb{T} \rightarrow \mathbb{H}$ is almost automorphic if from every sequence $\left\{s_{n}\right\}_{n=1}^{\infty} \subset$ $\Pi$, we can extract a subsequence $\left\{\tau_{n}\right\}_{n=1}^{\infty}$ such that :

$$
g(t)=\lim _{n \rightarrow \infty} f\left(t+\tau_{n}\right)
$$

is well defined for each $t \in \mathbb{T}$ and

$$
\lim _{n \rightarrow \infty} g\left(t-\tau_{n}\right)=f(t)
$$

for each $t \in \mathbb{T}$. Denote by $A A(\mathbb{T}, \mathbb{H})$ the set of all such functions.

Definition 2.11 ( [12]). Let $t_{0} \in \mathbb{T}$ and $r \in \Pi$. A function $f \in B C(\mathbb{T}, \mathbb{H})$, is said to be ergodic if

$$
\lim _{r \rightarrow+\infty} \frac{1}{2 r} \int_{t_{0}-r}^{t_{0}+r}\|f(s)\|_{\mathbb{H}} \Delta s=0,
$$

The space of all such functions is denoted by $P A A_{0}(\mathbb{T}, \mathbb{H})$.

Definition 2.12 ( [12]). A functions $f \in B C(\mathbb{T}, \mathbb{H})$ is called pseudo almost automorphic if $f=g+h$ where $g \in A A(\mathbb{T}, \mathbb{H})$ and $h \in P A A_{0}(\mathbb{T}, \mathbb{H})$.

We set,

$$
K= \begin{cases}\inf \{|\tau| ; \tau \in \mathbb{T}, \tau \neq 0\}, & \text { if } \mathbb{T} \neq \mathbb{R} \\ 1, & \text { if } \mathbb{T}=\mathbb{R}\end{cases}
$$

Let $f \in L_{l o c}^{p}(\mathbb{T}, \mathbb{H})$, for $1 \leq p<\infty$. Define :

- $\|\cdot\|_{S^{p}}: L_{l o c}^{p}(\mathbb{T}, \mathbb{H}) \rightarrow \mathbb{R}^{+}$as : $\|f\|_{S^{p}}=\sup _{t \in \mathbb{T}}\left(\frac{1}{K} \int_{t}^{t+K}|f(s)|_{\mathbb{H}}^{p} \Delta s\right)^{\frac{1}{p}}$.

- $C_{r d}(\mathbb{T} ; \mathbb{H})=\{f: \mathbb{T} \rightarrow \mathbb{H}: f$ is rd-continuous $\}$.

- $B C_{r d}(\mathbb{T} ; \mathbb{H})=\{f: T \rightarrow \mathbb{H}: f$ is bounded and rd-continuous $\}$.

- $L_{\text {loc }}^{p}(\mathbb{T} ; \mathbb{H})=\left\{f: \mathbb{T} \rightarrow \mathbb{H}: f\right.$ is locally $L^{p} \Delta$-integrable $\}$.

- $B S^{p}(\mathbb{T} ; \mathbb{H})=\left\{f \in L_{l o c}^{p}(\mathbb{T} ; \mathbb{H}):\|f\|_{S^{p}}<\infty\right\}$.

Definition 2.13 ( [12]). Let $f \in B S^{p}(\mathbb{T}, \mathbb{H})$ and $F \in B S^{p}(\mathbb{T} \times \mathbb{H}, \mathbb{H})$.

1) We say that $f: \mathbb{T} \rightarrow \mathbb{H}$ is Stepanov-like almost automorphic if for every sequence $\left\{s_{n}\right\}_{n=1}^{\infty} \subset \Pi$, we can extract a subsequence $\left\{\tau_{n}\right\}_{n=1}^{\infty}$ such that

$$
\left\|g(t)-f\left(t+\tau_{n}\right)\right\|_{S^{p}} \rightarrow 0, \quad \text { as } \quad n \rightarrow \infty
$$


is well defined for each $t \in \mathbb{T}$ and

$$
\left\|g\left(t-\tau_{n}\right)-f(t)\right\|_{S^{p}} \rightarrow 0, \quad \text { as } \quad n \rightarrow \infty,
$$

for each $t \in \mathbb{T}$. Denote by $S^{p} A A(\mathbb{T}, \mathbb{H})$ the set of all such functions.

2) A function $F: \mathbb{T} \times \mathbb{H} \rightarrow \mathbb{H},(t, x) \rightarrow F(t, x)$ is said to be Stepanov-like almost automorphic if $t \rightarrow F(t, x)$ is Stepanov almost automorphic in $t \in \mathbb{T}$ uniformly for each $x \in \mathbb{H}$. Denote by $S^{p} A A(\mathbb{T} \times \mathbb{H}, \mathbb{H})$ the collection of such functions.

Definition 2.14 ( [12]). A function $f \in B S^{p}(\mathbb{T}, \mathbb{H})$ is said to be Stepanov-like ergodic on $\mathbb{T}$ if :

$$
\lim _{r \rightarrow+\infty} \frac{1}{2 r} \int_{t_{0}-r}^{t_{0}+r}\left(\frac{1}{K} \int_{t}^{t+K}|f(s)|_{\mathbb{H}}^{p} \Delta s\right)^{\frac{1}{p}} \Delta t=0 .
$$

The space of all such functions will be denoted by $S^{p} P A A_{0}(\mathbb{T}, \mathbb{H})$.

Definition 2.15 ( [12]). A function $f \in B S^{p}(\mathbb{T}, \mathbb{H})$ is said to be Stepanov-like pseudo almost automorphic on $\mathbb{T}$ or briefly $S^{p}$ pseudo almost periodic if $f$ is written in the following form :

$$
f=g+\phi,
$$

where $g \in S^{p} A P(\mathbb{T}, \mathbb{H})$ and $\phi \in S^{p} P A A_{0}(\mathbb{T}, \mathbb{H})$. The space of all such functions will be denoted by $S^{p} P A A(\mathbb{T}, \mathbb{H})$.

Definition 2.16 ( [12]). A function $f: \mathbb{T} \times \mathbb{H} \rightarrow \mathbb{H}$ such that $f(., u) \in B S^{p}(\mathbb{T}, \mathbb{H})$ for each $u \in \mathbb{H}$ is said to be Stepanov-like pseudo almost automorphic if $f$ is written in the following form:

$$
f=g+\phi,
$$

where $g \in S^{p} A P(\mathbb{T} \times \mathbb{H}, \mathbb{H})$ and $\phi \in S^{p} P A A_{0}(\mathbb{T} \times \mathbb{H}, \mathbb{H})$. The space of all such functions will be denoted by $S^{p} P A A(\mathbb{T} \times \mathbb{H}, \mathbb{H})$.

Next, we recall the Bochner-like transform for general time scales.

If $\mathbb{T} \neq \mathbb{R}$, we fix a left scattered point $\omega \in \mathbb{T}$, there is a unique $n_{t} \in \mathbb{Z}$ such that $t-n_{t} K \in[\omega, \omega+k)_{\mathbb{T}}$. Let

$$
N_{t}= \begin{cases}t, & \mathbb{T}=\mathbb{R}, \\ n_{t} & \mathbb{T} \neq \mathbb{R} .\end{cases}
$$

Definition 2.17 ( $[10])$. Let $f \in B S^{p}(\mathbb{T}, \mathbb{H})$. The Bochner-like transform of $f$ is the function $f^{c}$ : $\mathbb{T} \times \mathbb{T} \rightarrow \mathbb{H}$ defined for all $t, s \in \mathbb{T}$ by

$$
f^{c}(t, s)=f\left(N_{t} K+s\right) .
$$


And we have

$$
\|f\|_{S^{p}}=\left\|f^{c}\right\|_{\infty}
$$

Definition 2.18 ( [12]). A function $f \in B S^{p}(\mathbb{T}, \mathbb{H})$ is said to be Stepanov-like pseudo almost automorphic if its Bochner-like transform $f^{c}$ is pseudo almost automorphic in the sense that there exist two functions $g, \phi$ such that $f^{c}=g^{c}+\phi^{c}$, where $g^{c} \in A A\left(\mathbb{T}, B S^{p}(\mathbb{T}, \mathbb{H})\right)$ and $\phi^{c} \in P A A_{0}\left(\mathbb{T}, B S^{p}(\mathbb{T}, \mathbb{H})\right)$.

Lemma 2.4 ( [12]). $\quad$ 1) If $h, g \in S^{p} P A A(\mathbb{T}, \mathbb{H})$, then $h+g, h g \in S^{p} P A A(\mathbb{T}$ $, \mathbb{H I})$.

2) If $h \in S^{p} P A A(\mathbb{T}, \mathbb{H})$ and $g \in S^{p} A P(\mathbb{T}, \mathbb{H})$, then $h g \in S^{p} P A A(\mathbb{T}, \mathbb{H})$.

Proposition 2.1 ( $[12])$. $\left(S^{p} P A A(\mathbb{T}, \mathbb{H}),\|\cdot\|_{S^{p}}\right)$ is a Banach space.

\section{Main Results}

3.1. Existence of $S^{p}$-pseudo almost automorphic solution of (2.1) on $\mathbb{T}$. In this section, we will study the existence and $S^{p}$-global exponential stability of Stepanov-like pseudo almost automorphic solution of system (2.1) on time scales.

We will list a few hypotheses which will be used for the rest of this paper.

$\left(S_{1}\right):$ For all $1 \leq l \leq n$, the function $\xi_{m}(.) \in S^{p} A A(\mathbb{T}, \mathbb{H}) \cap C_{r d}^{1}(\mathbb{T}, \mathbb{H})$ such that

$$
0 \leq \xi_{m}(.) \leq \bar{\xi}, \quad 0 \leq \xi^{*}-\xi_{m}^{\Delta}(.)<1-\xi_{m}^{\Delta}(.)
$$

$\left(S_{2}\right):$ For all $1 \leq l, m \leq n$, the functions $a_{l m}(),. b_{l m}(),. c_{l m}(),. d_{l m}(.) \in S^{p} P A A(\mathbb{T}, \mathbb{H})$.

$\left(S_{3}\right)$ : There exist positive constants $L_{l}^{f}, L_{l}^{g}, L_{h_{l}}$ such that for any $u, v \in \mathbb{H}$, the activity functions $f_{l}$, $g_{l}, h_{l} \in C_{r d}(\mathbb{H}, \mathbb{H})$ satisfy

$$
\begin{aligned}
& \left|f_{l}(u)-f_{l}(v)\right|_{\mathbb{H}} \leq L_{l}^{f}|u-v|_{\mathbb{H}}, \\
& \left|g_{l}(u)-g_{l}(v)\right|_{\mathbb{H}} \leq L_{l}^{g}|u-v|_{\mathbb{H}}, \\
& \left|h_{l}(u)-h_{l}(v)\right|_{\mathbb{H}} \leq L_{l}^{h}|u-v|_{\mathbb{H}} .
\end{aligned}
$$

Furthermore, we suppose that $f_{l}(0)=g_{l}(0)=h_{l}(0)=0$.

$\left(S_{4}\right)$ : For all $1 \leq l, m \leq n$, the delay kernel $N_{l m}:[0,+\infty)_{\mathbb{T}} \rightarrow[0,+\infty)$ is rd-continuous, $\Delta$-integrable and there exists $\lambda>0$ such that $\int_{0}^{+\infty} N_{l m}(z) \Delta z=N^{*}$.

$\left(S_{5}\right): \varpi<1$.

As a convenience, we have introduced these notations which simplify the writing of the equations:

$$
b_{l m}^{*}=\sup _{t \in \mathbb{T}}\left|b_{l m}(t)\right|_{\mathbb{H}}, \quad c_{l m}^{*}=\sup _{t \in \mathbb{T}}\left|c_{l m}(t)\right|_{\mathbb{H}}, \quad d_{l m}^{*}=\sup _{t \in \mathbb{T}}\left|d_{l m}(t)\right|_{\mathbb{H}}, \quad \bar{\mu}=\sup _{t \in \mathbb{T}} \mu(t)
$$




$$
\begin{gathered}
\bar{a}_{l}=\inf _{t \in \mathbb{T}} a_{l}(t) \quad a_{l}^{*}=\sup _{t \in \mathbb{T}} a_{l}(t)>0 \quad \check{a}_{l}=\inf _{t \in \mathbb{T}} \bar{a}_{l}+\inf _{t \in \mathbb{T}} a_{l} \quad l=1, \ldots, n, \\
\varpi=\max _{1 \leq l \leq n}\left\{r_{l}^{\frac{1}{q}}(q) \cdot r_{l}^{\frac{1}{p}}(p) \times\left[\sum_{m=1}^{n} b_{l m}^{*} L_{m}^{f}+\sum_{m=1}^{n} \frac{c_{l m}^{*} L_{m}^{g}}{\left(1-\xi_{m}^{*}\right)^{\frac{1}{p}}}+\sum_{m=1}^{n} d_{l m}^{*} L_{m}^{h} N^{*}\right]\right\}, \\
\text { with } r_{l}(q)=\frac{2+\bar{a}_{l} \bar{\mu} q}{\bar{a}_{l} q}, \quad \rho_{I}=\max _{l=1, \ldots, n} r_{l}^{\frac{1}{q}}(q) r_{l}^{\frac{1}{p}}(p)\|I\|_{S^{p}}, \quad r^{*}=\frac{\varpi \rho_{I}}{1-\varpi} .
\end{gathered}
$$

Lemma 3.1. Suppose that condition $\left(S_{1}\right)$ holds. If $g \in S^{p} P A A(\mathbb{T}, \mathbb{H})$ and $\xi \in S^{p} A A(\mathbb{T}, \mathbb{H})$, then $g(.-\xi().) \in S^{p} P A A(\mathbb{T}, \mathbb{H})$.

Proof. Since $f \in S^{p} P A A(\mathbb{T}, \mathbb{H})$, then,

$$
g(t-\xi(t))=g_{1}(t-\xi(t))+g_{2}(t-\xi(t)):=G_{1}(t)+G_{2}(t), \quad \forall t \in \mathbb{T}
$$

where $g_{1} \in S^{p} A A(\mathbb{T}, \mathbb{H})$ and $g_{2} \in S^{p} P A A_{0}(\mathbb{T}, \mathbb{H})$. It's obvious that $G_{1}(t)=g_{1}(t-\xi(t)) \in S^{p} A A(\mathbb{T}, \mathbb{H})$. It remains to show that $G_{2}()=.g_{2}(.-\xi().) \in S^{p} P A A_{0}(\mathbb{T}, \mathbb{H})$. Since $g_{2} \in S^{p} P A A_{0}(\mathbb{T}, \mathbb{H})$, we have for all $t \in \mathbb{T}$,

$$
F(t)=\left(\frac{1}{K} \int_{t}^{t+K}\left|g_{2}(s)\right|_{\mathbb{H}}^{p} \Delta s\right)^{\frac{1}{p}} \in S^{p} P A A_{0}(\mathbb{T}, \mathbb{H})
$$

Thus,

$$
\begin{aligned}
\lim _{r \rightarrow+\infty} & \frac{1}{2 r} \int_{t_{0}-r}^{t_{0}+r}\left(\frac{1}{K} \int_{t}^{t+K}\left|g_{2}(s-\xi(s))\right|_{\mathbb{H}}^{p} \Delta s\right)^{\frac{1}{p}} \Delta t \\
& =\frac{1}{\left(1-\xi^{\Delta}(s)\right)^{\frac{1}{p}}} \lim _{r \rightarrow+\infty} \frac{1}{2 r} \int_{t_{0}-r}^{t_{0}+r}\left(\frac{1}{K} \int_{t-\xi(t)}^{t+K-\xi(t+k)}\left|g_{2}(z)\right|_{\mathbb{H}}^{p} \Delta z\right)^{\frac{1}{p}} \Delta t \\
& \leq \frac{1}{\left(1-\xi^{*}\right)^{\frac{1}{p}}} \lim _{r \rightarrow+\infty} \frac{1}{2 r}\left(\frac{1}{K} \int_{t-\bar{\xi}}^{t+K-\bar{\xi}}\left|g_{2}(z)\right|_{\mathbb{H}}^{p} \Delta z\right)^{\frac{1}{p}} \Delta t \\
& \leq \frac{1}{\left(1-\xi^{*}\right)^{\frac{1}{p}}} \lim _{r \rightarrow+\infty} \frac{1}{2 r} \int_{t_{0}-r}^{t_{0}+r}|F(t-\bar{\xi})|_{\mathbb{H}} \Delta t, \\
& \leq \frac{1}{\left(1-\xi^{*}\right)^{\frac{1}{p}}} \lim _{r \rightarrow+\infty} \frac{1}{2 r} \int_{t_{0}-r-\bar{\xi}}^{t_{0}+r-\bar{\xi}}|F(t)|_{\mathbb{H}} \Delta t \\
& \leq \frac{1}{\left(1-\xi^{*}\right)^{\frac{1}{p}}} \lim _{r \rightarrow+\infty} \frac{1}{2 r} \int_{t_{0}-r-\bar{\xi}}^{t_{0}+r+\bar{\xi}}|F(t)|_{\mathbb{H}} \Delta t \\
= & \frac{1}{\left(1-\xi^{*}\right)^{\frac{1}{p}}} \lim _{r \rightarrow+\infty} \frac{r+\bar{\xi}}{r} \frac{1}{2(r+\bar{\xi})} \int_{t_{0}-r-\bar{\xi}}^{t_{0}+r+\bar{\xi}}|F(t)|_{\mathbb{H}} \Delta t \\
= & 0 .
\end{aligned}
$$

So, $G_{2} \in S^{p} P A A_{0}(\mathbb{T}, \mathbb{H})$. 
Lemma 3.2. If a function $g \in C_{r d}(\mathbb{H}, \mathbb{H})$ satisfies condition $\left(S_{4}\right)$ and $x \in S^{p} P A A(\mathbb{T}, \mathbb{H})$, then $g \circ x \in$ $S^{p} P A A(\mathbb{T}, \mathbb{H})$.

Proof. We have $x \in S^{p} P A A(\mathbb{T}, \mathbb{H})$, then $x=x_{1}+x_{2}$ where $x_{1} \in S^{p} A A(\mathbb{T}, \mathbb{H})$ and $x_{2} \in S^{p} P A A_{0}(\mathbb{T}, \mathbb{H})$. Hence,

$$
g \circ x(.)=g \circ x_{1}(.)+g \circ x(.)-g \circ x_{1}(.)=G_{1}(.)+G_{2}(.),
$$

where, $G_{1}()=.g \circ x_{1}($.$) and G_{2}()=.g \circ x()-.g \circ x_{1}($.$) . Since x_{1} \in S^{p} A A(\mathbb{T}, \mathbb{H})$, then for every sequence $\left\{s_{n}\right\}_{n=1}^{\infty} \subset \Pi$, we can extract a subsequence $\left\{\tau_{n}\right\}_{n=1}^{\infty}$ such that

$$
\sup _{t \in \mathbb{T}}\left(\frac{1}{K} \int_{t}^{t+K}\left|x_{1}\left(s+\tau_{n}\right)-\varphi(s)\right|_{\mathbb{H}}^{p} \Delta s\right)^{\frac{1}{p}} \rightarrow 0, \quad \text { as } \quad n \rightarrow \infty .
$$

We set $\varphi_{1}():.=g \circ \varphi$, therefore,

$$
\begin{aligned}
\sup _{t \in \mathbb{T}}\left(\frac{1}{K} \int_{t}^{t+K}\left|g \circ x_{1}\left(s+\tau_{n}\right)-\varphi_{1}(s)\right|_{\mathbb{H}}^{p} \Delta s\right)^{\frac{1}{p}} \\
\quad=\sup _{t \in \mathbb{T}}\left(\frac{1}{K} \int_{t}^{t+K}\left|g \circ x_{1}\left(s+\tau_{n}\right)-g \circ \varphi(s)\right|_{\mathbb{H}}^{p} \Delta s\right)^{\frac{1}{p}}, \\
\leq L^{g} \sup _{t \in \mathbb{T}}\left(\frac{1}{K} \int_{t}^{t+K}\left|x_{1}\left(s+\tau_{n}\right)-\varphi(s)\right|_{\mathbb{H}}^{p} \Delta s\right)^{\frac{1}{p}} .
\end{aligned}
$$

Then,

$$
\sup _{t \in \mathbb{T}}\left(\frac{1}{K} \int_{t}^{t+K}\left|g \circ x_{1}\left(s+\tau_{n}\right)-\varphi_{1}(s)\right|_{\mathbb{H}}^{p} \Delta s\right)^{\frac{1}{p}} \rightarrow 0, \quad \text { as } \quad n \rightarrow \infty .
$$

Because of that $x_{1}(.) \in S^{p} A A(\mathbb{T}, \mathbb{H})$. Now,

$$
\begin{aligned}
\lim _{r \rightarrow+\infty} & \frac{1}{2 r} \int_{t_{0}-r}^{t_{0}+r}\left(\frac{1}{K} \int_{t}^{t+K}\left|G_{2}(s)\right|_{\mathbb{H}}^{p} \Delta s\right)^{\frac{1}{p}} \Delta t \\
& =\lim _{r \rightarrow+\infty} \frac{1}{2 r} \int_{t_{0}-r}^{t_{0}+r}\left(\frac{1}{K} \int_{t}^{t+K}\left|g \circ x(s)-g \circ x_{1}(s)\right|_{\mathbb{H}}^{p} \Delta s\right)^{\frac{1}{p}} \Delta t \\
& \leq \lim _{r \rightarrow+\infty} \frac{L^{g}}{2 r} \int_{t_{0}-r}^{t_{0}+r}\left(\frac{1}{K} \int_{t}^{t+K}\left|x(s)-x_{1}(s)\right|_{\mathbb{H}}^{p} \Delta s\right)^{\frac{1}{p}} \Delta t \\
& \leq \lim _{r \rightarrow+\infty} \frac{L^{g}}{2 r} \int_{t_{0}-r}^{t_{0}+r}\left(\frac{1}{K} \int_{t}^{t+K}\left|x_{2}(s)\right|_{\mathbb{H}}^{p} \Delta s\right)^{\frac{1}{p}} \Delta t \\
& =0
\end{aligned}
$$

As a consequence, $G_{2}(.) \in S^{p} P A A_{0}(\mathbb{T}, \mathbb{H})$. In conclusion, $g \circ x \in S^{p} P A A(\mathbb{T}, \mathbb{H})$.

Lemma 3.3. Assume that $\left(S_{3}\right)$ and $\left(S_{4}\right)$ hold and $x(.) \in S^{p} P A A(\mathbb{T}, \mathbb{H})$, then for $l, m=1,2, \ldots, n$, the function $t \rightarrow \int_{-\infty}^{t} N_{l m}(t-s) h_{m}\left(x_{m}(s)\right) \Delta$ s belongs to $S^{p} P A A(\mathbb{T}, \mathbb{H})$, for all $t, s \in \mathbb{T}$. 
Proof. We have $x_{m} \in S^{p} P A A(\mathbb{T}, \mathbb{H})$. Then,

$$
\begin{aligned}
\left|\phi_{l m}(t)\right|_{\mathbb{H}} & \leq \int_{-\infty}^{t}\left|N_{l m}(t-s) h_{m}\left(x_{m}(s)\right) \Delta s\right|_{\mathbb{H}} \\
& \leq L_{m}^{h}\left\|x_{m}\right\|_{S^{p}} \int_{0}^{+\infty} \mid N_{l m}(s) \Delta s \\
& \leq N^{*} L_{m}^{h}\left\|x_{m}\right\|_{S^{p}} .
\end{aligned}
$$

which proves that the integral $\int_{-\infty}^{t} N_{l m}(t-s) h_{m}\left(x_{m}(s)\right) \Delta s$ is absolutely convergent and $\phi_{l m} \in B S^{p}(\mathbb{T}, \mathbb{H})$. Hence, it is easy to prove the rd-continuity of function $\phi_{l m}$. Now, from lemma (3.2) we have, $h_{m}^{c}\left(x_{m}^{c}().\right) \in$ $P A A\left(\mathbb{T}, B S^{p}(\mathbb{T}, \mathbb{H})\right)$. Then, for all $1 \leq m \leq n$, we get

$$
h_{m}^{c}\left(x_{m}^{c}(.)\right)=y_{m}^{c}(.)+z_{m}^{c}(.)
$$

Where $y_{m}^{c}(.) \in A A\left(\mathbb{T}, B S^{p}(\mathbb{T}, \mathbb{H})\right)$ and $z_{m}^{c}(.) \in P A A_{0}\left(\mathbb{T}, B S^{p}(\mathbb{T}, \mathbb{H})\right)$. Thus, for all $1 \leq m, l \leq n$, we can write $\phi_{l m}$ in the following form

$$
\begin{aligned}
\phi_{l m}^{c}(t) & =\int_{-\infty}^{t} N_{l m}(t-s) y_{m}^{c}(s) \Delta s+\int_{-\infty}^{t} N_{l m}(t-s) z_{m}^{c}(s) \Delta s \\
& =Y_{l m}^{c}(t)+Z_{l m}^{c}(t) .
\end{aligned}
$$

On the one hand, let us prove that $Y_{l m}^{c}(.) \in A A\left(\mathbb{T}, B S^{p}(\mathbb{T}, \mathbb{H})\right)$. Since $y_{m}^{c}(.) \in A A\left(\mathbb{T}, B S^{p}(\mathbb{T}, \mathbb{H})\right)$, so for every sequence $\left\{s_{n}\right\}_{n=1}^{\infty} \subset \Pi$, we can extract a subsequence $\left\{\tau_{n}\right\}_{n=1}^{\infty}$ such that

$$
\lim _{n \rightarrow \infty} y_{m}^{c}\left(t+\tau_{n}\right)=\varphi_{m}(t) \quad \text { and } \quad y_{m}^{c}(t)=\lim _{n \rightarrow \infty} \varphi_{m}\left(t-\tau_{n}\right) .
$$

We pose $W_{l m}:=\int_{-\infty}^{t} N_{l m}(t-s) \varphi_{m}(s) \Delta s$. Then we have

$$
\begin{aligned}
\| Y_{l m}\left(t+\tau_{n}\right)-W_{l m}(t) & \left\|_{S^{p}}=\right\| Y_{l m}^{c}\left(t+\tau_{n}\right)-W_{l m}(t) \|_{\infty} \\
& =\| \int_{-\infty}^{t+\tau_{n}} N_{l m}\left(t+\tau_{n}-s\right) y_{m}^{c}(s) \Delta s \\
& -\int_{-\infty}^{t} N_{l m}(t-s) \varphi_{m}(s) \Delta s \|_{\infty} \\
& \leq \int_{-\infty}^{t} N_{l m}(t-s)\left\|y_{m}^{c}\left(s+\tau_{n}\right)-\varphi_{m}(s)\right\|_{\infty} \Delta s .
\end{aligned}
$$

By the Lebesgue dominated convergence theorem, we obtain

$$
\lim _{n \rightarrow \infty} Y_{m}^{c}\left(t+\tau_{n}\right)=W_{l m}(t) \quad \forall t \in \mathbb{T} .
$$

Similarly, we can obtain

$$
Y_{m}^{c}(t)=\lim _{n \rightarrow \infty} W_{l m}\left(t-\tau_{n}\right) \quad \forall t \in \mathbb{T} .
$$


As a result, $Y_{l m}^{c}(.) \in A A\left(\mathbb{T}, B S^{p}(\mathbb{T}, \mathbb{H})\right)$.

On the other hand, it remains to show that $Z_{l m}^{c}(.) \in P A A_{0}\left(\mathbb{T}, B S^{p}(\mathbb{T}, \mathbb{H})\right)$. Indeed,

$$
\begin{aligned}
\lim _{r \rightarrow \infty} \frac{1}{2 r} \int_{t_{0}-r}^{t_{0}+r} \| Z_{l m}(s) & \left\|_{S^{p}} \Delta t=\lim _{r \rightarrow \infty} \frac{1}{2 r} \int_{t_{0}-r}^{t_{0}+r}\right\| Z_{l m}^{c}(s) \|_{\infty} \Delta t \\
& \leq \lim _{r \rightarrow \infty} \frac{1}{2 r} \int_{t_{0}-r}^{t_{0}+r} \int_{0}^{+\infty} N_{l m}(s)\left\|z_{m}^{c}(t-s)\right\|_{\infty} \Delta s \Delta t \\
& \leq \int_{0}^{+\infty} N_{l m}(s) \lim _{r \rightarrow \infty} \frac{1}{2 r} \int_{t_{0}-r}^{t_{0}+r}\left\|z_{m}^{c}(t-s)\right\|_{\infty} \Delta t \Delta s \\
& =0
\end{aligned}
$$

Thus, $Y_{l m}^{c}(.) \in P A A_{0}\left(\mathbb{T}, B S^{p}(\mathbb{T}, \mathbb{H})\right)$, then, $\phi_{l m}^{c}(.) \in P A A\left(\mathbb{T}, B S^{p}(\mathbb{T}, \mathbb{H})\right)$. Finally, the function $\phi_{l m}$ belongs to $S^{p} P A A(\mathbb{T}, \mathbb{H})$.

Theorem 3.1. Let $\psi=\left(\psi_{1}, \ldots, \psi_{n}\right) \in S^{p} P A A(\mathbb{T}, \mathbb{H})$. Under assumptions $\left(S_{1}\right)-\left(S_{4}\right)$, the nonlinear operator defined by,

$$
\left(\Pi_{\psi}\right)_{l}(t)=\int_{-\infty}^{t} \hat{e}_{\ominus a_{l}}(t, \sigma(s)) J_{l}(s) \Delta s, \quad l=1, \ldots, n
$$

Where

$$
\begin{aligned}
J_{l}(t) & =\sum_{m=1}^{n} b_{l m}(t) f_{m}\left(\psi_{m}(t)\right)+\sum_{m=1}^{n} c_{l m}(t) g_{m}\left(\psi_{m}\left(t-\xi_{m}(t)\right)\right) \\
& +\sum_{m=1}^{n} d_{l m}(t) \int_{-\infty}^{t} N_{l m}(t-s) h_{m}\left(\psi_{m}(s)\right) \Delta s+I_{l}(t)
\end{aligned}
$$

maps $S^{p} P A A(\mathbb{T}, \mathbb{H})$ into itself.

Proof. It's easy to see that $\left(\Pi_{\psi}\right)_{l}$ is well defined and continuous. Again from lemma (3.1) and Lemma (3.3), we have $J_{l}($.$) belongs to S^{p} P A A(\mathbb{T}, \mathbb{H})$. Now, let $J_{l}=Y_{l}+Z_{l}$ with $Y_{l} \in S^{p} A A(\mathbb{T}, \mathbb{H})$, $Z_{l}(.) \in S^{p} P A A_{0}(\mathbb{T}, \mathbb{H})$. For the sake of convenience, we break the proof in two steps.

First : We will prove that $\left(F_{\psi}\right)_{l}(t)=\int_{-\infty}^{t} \hat{e}_{\ominus a_{l}}(t, \sigma(s)) Y_{l}(s) \Delta s \in S^{p} A A(\mathbb{T}, \mathbb{H})$. Since $Y_{l}(.) \in S^{P} A A(\mathbb{T}, \mathbb{H})$, then for every sequence $\left\{s_{n}\right\}_{n=1}^{\infty} \subset \Pi$, we can extract a subsequence $\left\{\tau_{n}\right\}_{n=1}^{\infty}$ such that

$$
\sup _{t_{1} \in \mathbb{T}}\left(\frac{1}{K} \int_{t_{1}}^{t_{1}+K}\left|Y_{l}\left(t+\tau_{n}\right)-\varphi_{l}(t)\right|_{\mathbb{H}}^{p} \Delta t\right)^{\frac{1}{p}} \rightarrow 0, \quad \text { as } \quad n \rightarrow \infty
$$


We set $\left(\mathscr{U}_{\psi}\right)_{l}(t)=\int_{-\infty}^{t} \hat{e}_{\ominus a_{l}}(t, \sigma(s)) \varphi_{l}(s) \Delta s$. Thus,

$$
\begin{aligned}
& \left|\left(F_{\psi}\right)_{l}\left(t+\tau_{n}\right)-\left(\mathscr{U}_{\psi}\right)_{l}(t)\right|_{\mathbb{H}} \\
& \quad=\left|\int_{-\infty}^{t} \hat{e}_{\ominus a_{l}}(t, \sigma(s)) Y_{l}\left(s+\tau_{n}\right) \Delta s-\int_{-\infty}^{t} \hat{e}_{\ominus a_{l}}(t, \sigma(s)) \varphi_{l}(s) \Delta s\right|_{\mathbb{H}}, \\
& \leq \int_{-\infty}^{t} \hat{e}_{\ominus \bar{a}_{l}}(t, \sigma(s))\left|Y_{l}\left(s+\tau_{n}\right)-\varphi_{l}(s)\right|_{\mathbb{H}} \Delta s, \\
& \leq \int_{-\infty}^{0} \hat{e}_{\ominus \bar{a}_{l}}(0, \sigma(s))\left|Y_{l}\left(t+s+\tau_{n}\right)-\varphi_{l}(t+s)\right|_{\mathbb{H}} \Delta s, \\
& \leq r_{l}^{\frac{1}{q}}(q)\left(\int_{-\infty}^{0} \hat{e}_{\ominus\left(\frac{\bar{l}_{l} p}{2}\right)}(0, \sigma(s))\left|Y_{l}\left(t+s+\tau_{n}\right)-\varphi_{l}(t+s)\right|_{\mathbb{H}}^{p} \Delta s\right)^{\frac{1}{p}} .
\end{aligned}
$$

Fubini's theorem implies that

$$
\begin{aligned}
& \sup _{t_{1} \in \mathbb{T}}\left(\frac{1}{K} \int_{t_{1}}^{t_{1}+K}\left|\left(F_{\psi}\right)_{l}\left(t+\tau_{n}\right)-\left(\mathscr{U}_{\psi}\right)_{l}(t)\right|_{\mathbb{H}}^{p} \Delta t\right)^{\frac{1}{p}} \\
& \leq \sup _{t_{1} \in \mathbb{T}}\left(\frac{1}{K} \int_{t_{1}}^{t_{1}+K} r_{l}^{\frac{p}{q}} \cdot \int_{-\infty}^{0} \hat{e}_{\ominus\left(\frac{\bar{a}_{l} p}{2}\right)}(0, \sigma(s))\left|Y_{l}\left(s+t+\tau_{n}\right)-\varphi_{l}(s+t)\right|_{\mathbb{H}}^{p} \Delta s \Delta t\right)^{\frac{1}{p}}, \\
& \leq r_{l}^{\frac{1}{q}}(q) \cdot\left(\int_{-\infty}^{0} \hat{e}_{\ominus\left(\frac{\bar{a}_{l} p}{2}\right)}(0, \sigma(s)) \sup _{\bar{t} \in \mathbb{T}} \frac{1}{K} \int_{\bar{t}}^{\bar{t}+K}\left|Y_{l}\left(t+\tau_{n}\right)-\varphi_{l}(t)\right|_{\mathbb{H}}^{p} \Delta t \Delta s\right)^{\frac{1}{p}}, \\
& \leq r_{l}^{\frac{1}{q}}(q) \cdot r_{l}^{\frac{1}{p}}(p) \cdot\left(\sup _{\bar{t} \in \mathbb{T}} \frac{1}{K} \int_{\bar{t}}^{\bar{t}+K}\left|Y_{l}\left(t+\tau_{n}\right)-\varphi_{l}(t)\right|_{\mathbb{H}}^{p} \Delta t \Delta s\right)^{\frac{1}{p}} \\
& <\max _{1 \leq l \leq n} r_{l}^{\frac{1}{q}}(q) r_{l}^{\frac{1}{p}}(p)\left(\sup _{\bar{t} \in \mathbb{T}} \frac{1}{K} \int_{\bar{t}}^{\bar{t}+K}\left|Y_{l}\left(t+\tau_{n}\right)-\varphi_{l}(t)\right|_{\mathbb{H}}^{p} \Delta t \Delta s\right)^{\frac{1}{p}} .
\end{aligned}
$$

Therefore,

$$
\sup _{t_{1} \in \mathbb{T}}\left(\frac{1}{K} \int_{t_{1}}^{t_{1}+K}\left|\left(F_{\psi}\right)_{l}\left(t+\tau_{n}\right)-\left(\mathscr{U}_{\psi}\right)_{l}(t)\right|_{\mathbb{H}}^{p} \Delta t\right)^{\frac{1}{p}} \rightarrow 0, \quad \text { as } \quad n \rightarrow \infty .
$$

Which means that $\left(F_{\psi}\right)_{l}(.) \in S^{p} A A(\mathbb{T}, \mathbb{H})$.

Second : Let us prove that $\left(G_{\psi}\right)_{l}(t)=\int_{-\infty}^{t} \hat{e}_{\ominus a_{l}}(t, \sigma(s)) Z_{l}(s) \Delta s \in S^{p} P A A_{0}(\mathbb{T}, \mathbb{H})$. It follows from Hölder's inequality and Fubini's theorem that

$$
\begin{aligned}
& \int_{t_{0}-r}^{t_{0}+r}\left(\frac{1}{K} \int_{t}^{t+K}\left|\left(G_{\psi}\right)_{l}(s)\right|_{\mathbb{H}}^{p} \Delta s\right)^{\frac{1}{p}} \Delta t \leq \\
& (2 r)^{\frac{1}{q}}\left[\int_{t_{0}-r}^{t_{0}+r}\left(\frac{1}{K} \int_{t}^{t+K}\left|\left(G_{\psi}\right)_{l}(s)\right|_{\mathbb{H}}^{p} \Delta s\right) \Delta t\right]^{\frac{1}{p}} .
\end{aligned}
$$


Since,

$$
\begin{aligned}
& \int_{t_{0}-r}^{t_{0}+r}\left(\frac{1}{K} \int_{t}^{t+K}\left|\left(G_{\psi}\right)_{l}(s)\right|_{\mathbb{H}}^{p} \Delta s\right)^{\frac{1}{p}} \Delta t \\
& \leq(2 r)^{\frac{1}{q}}\left[\int_{t_{0}-r}^{t_{0}+r}\left(\frac{1}{K} \int_{t}^{t+K} r_{l}^{\frac{p}{q}}(q) \int_{-\infty}^{0} \hat{e}_{\ominus\left(\frac{\bar{a}_{l} p}{2}\right)}(0, \sigma(z))\left|Z_{m}(s+z)\right|_{\mathbb{H}}^{p} \Delta z \Delta s\right) \Delta t\right]^{\frac{1}{p}}, \\
& \leq(2 r)^{\frac{1}{q}} r_{l}^{\frac{1}{q}}(q)\left[\int_{-\infty}^{0} \hat{e}_{e\left(\frac{\bar{a}_{l} p}{2}\right)}(0, \sigma(z)) \int_{t_{0}-r}^{t_{0}+r} \frac{1}{K} \int_{t}^{t+K}\left|Z_{l}(s+z)\right|_{\mathbb{H}}^{p} \Delta s \Delta t \Delta z\right]^{\frac{1}{p}} .
\end{aligned}
$$

According to Lebesgue dominated theorem and $Z_{l}(.) \in S^{p} P A A_{0}(\mathbb{T}, \mathbb{H})$, we get

$$
\begin{aligned}
& \lim _{r \rightarrow \infty} \frac{1}{2 r} \int_{t_{0}-r}^{t_{0}+r}\left(\frac{1}{K} \int_{t}^{t+K}\left|\left(G_{\psi}\right)_{l}(s)\right|_{\mathbb{H}}^{p} \Delta s\right)^{\frac{1}{p}} \Delta t \leq \frac{1}{2 r}(2 r)^{\frac{1}{q}}(2 r)^{\frac{1}{p}} r_{l}^{\frac{1}{q}}(q) \\
& \times\left(\int_{-\infty}^{0} \hat{e}_{\ominus\left(\frac{\bar{l}_{l} p}{2}\right)}(0, \sigma(z)) \lim _{r \rightarrow \infty} \frac{1}{2 r} \int_{t_{0}-r}^{t_{0}+r} \frac{1}{K} \int_{t}^{t+K}\left|Z_{l}(s+z)\right|_{\mathbb{H}}^{p} \Delta s \Delta t \Delta z\right)^{\frac{1}{p}}, \\
& \leq r_{l}^{\frac{1}{q}}(q)\left[\int_{-\infty}^{0} \hat{e}_{\ominus\left(\frac{\bar{l}_{l} p}{2}\right)}(0, \sigma(z)) \lim _{r \rightarrow \infty} \frac{1}{2 r} \int_{t_{0}-r}^{t_{0}+r} \frac{1}{K} \int_{t}^{t+K}\left|Z_{l}(s+z)\right|_{\mathbb{H}}^{p} \Delta s \Delta t \Delta z\right]^{\frac{1}{p}}, \\
& \leq r_{l}^{\frac{1}{q}}(q)\left[\int_{-\infty}^{0} \hat{e}_{\ominus\left(\frac{\bar{a}_{l} p}{2}\right)}(0, \sigma(z)) \lim _{r \rightarrow \infty} \frac{1}{2 r} \int_{t_{0}-r}^{t_{0}+r}\left(\frac{1}{K} \int_{t}^{t+K}\left|Z_{l}(s+z)\right|_{\mathbb{H}}^{q} \Delta s\right)^{\frac{p}{q}} \Delta t \Delta z\right]^{\frac{1}{p}}, \\
& \leq r_{l}^{\frac{1}{q}}(q)\left[\int_{-\infty}^{0} \hat{e}_{\ominus\left(\frac{\bar{a}_{l} p}{2}\right)}(0, \sigma(z)) \lim _{r \rightarrow \infty} \frac{1}{2 r} \int_{t_{0}-r}^{t_{0}+r}\left(\frac{1}{K} \int_{t}^{t+K}\left|Z_{l}(s+z)\right|_{\mathbb{H}}^{q} \Delta s\right)^{\frac{1}{q}}\right. \\
& \left.\times\left(\frac{1}{K} \int_{t}^{t+K}\left|Z_{l}(s+z)\right|_{\mathbb{H}}^{q} \Delta s\right)^{\frac{p-1}{q}} \Delta t \Delta z\right]^{\frac{1}{p}}, \\
& \leq r_{l}^{\frac{1}{q}}(q)\left|Z_{l}\right|_{\infty}^{\frac{p-1}{p}}\left\{\int_{-\infty}^{0} \hat{e}_{\ominus\left(\frac{\bar{a}_{l} p}{2}\right)}(0, \sigma(z))\right. \\
& \left.\times\left[\lim _{r \rightarrow \infty} \frac{1}{2 r} \int_{t_{0}-r}^{t_{0}+r}\left(\frac{1}{K} \int_{t}^{t+K}\left|Z_{l}(s+z)\right|_{\mathbb{H}}^{q} \Delta s\right)^{\frac{1}{q}} \Delta t\right] \Delta z\right\}^{\frac{1}{p}}=0 .
\end{aligned}
$$

Which yields that $\left(G_{\psi}\right)_{l}(.) \in S^{p} P A A_{0}(\mathbb{T}, \mathbb{H})$. This completes the proof of theorem.

Theorem 3.2. Assume that the conditions $\left(S_{1}\right)-\left(S_{5}\right)$ are satisfied. Then, system (2.1) has a unique $S^{p}$-pseudo almost automorphic solution in the region $\mathbb{B}=\left\{\psi: \psi \in S^{p} P A A(\mathbb{T}, \mathbb{H}),\left\|\psi-\psi_{0}\right\|_{S^{p}} \leq r^{*}\right\}$. 
Proof. First step : At first, we show that $\left(\Pi_{\psi}\right)_{l}$ is a self-mapping from $\mathbb{B}$ to $\mathbb{B}$. Let $\psi \in \mathbb{B}$, by using Hölder's and Minkowski's inequality we can obtain

$$
\begin{aligned}
& \left|\left(\Lambda_{\psi}\right)_{l}(t)-\psi_{0}(t)\right|_{\mathbb{H}}=\mid \int_{-\infty}^{t} \hat{e}_{\ominus a_{l}}(t, \sigma(z)) \times\left[\sum_{m=1}^{n} b_{l m}(z) f_{m}\left(\psi_{m}(z)\right)\right. \\
& \left.+\sum_{m=1}^{n} c_{l m}(z) g_{m}\left(\psi_{m}\left(z-\xi_{m}(z)\right)\right)+\sum_{m=1}^{n} d_{l m}(z) \int_{-\infty}^{z} N_{l m}(z-s) h_{m}\left(\psi_{m}(s)\right) \Delta s\right]\left.\Delta z\right|_{\mathbb{H}} \\
& \leq r_{l}^{\frac{1}{q}}(q) \times\left[\int_{-\infty}^{0} \hat{e}_{\ominus\left(\frac{\bar{a}_{l} p}{2}\right)}(0, \sigma(z)) \mid \sum_{m=1}^{n} b_{l m}(z+t) f_{m}\left(\psi_{m}(z+t)\right)\right. \\
& +\sum_{m=1}^{n} c_{l m}(z+t) g_{m}\left(\psi_{m}\left(z+t-\xi_{m}(z+t)\right)\right) \\
& \left.+\left.\sum_{m=1}^{n} d_{l m}(z+t) \int_{-\infty}^{z+t} N_{l m}(z+t-s) h_{m}\left(\psi_{m}(s)\right) \Delta s\right|_{\mathbb{H}} ^{p} \Delta z\right]^{\frac{1}{p}}
\end{aligned}
$$

Furthermore

$$
\begin{aligned}
& \left\|\left(\Pi_{\psi}\right)_{l}(t)-\psi_{0}(t)\right\|_{S^{p}}=\sup _{t_{1} \in \mathbb{T}}\left[\frac{1}{K} \int_{t_{1}}^{t_{1}+K} \mid \int_{-\infty}^{t} \hat{e}_{\ominus a_{l}}(t, \sigma(z))\right. \\
& \times\left(\sum_{m=1}^{n} b_{l m}(z) f_{m}\left(\psi_{m}(z)\right)+\sum_{m=1}^{n} c_{l m}(z) g_{m}\left(\psi_{m}\left(z-\xi_{m}(z)\right)\right)\right. \\
& \left.\left.+\sum_{m=1}^{n} d_{l m}(z) \int_{-\infty}^{z} N_{l m}(z-s) h_{m}\left(\psi_{m}(s)\right) \Delta s\right)\left.\Delta z\right|_{\mathbb{H}} ^{p} \Delta t\right]^{\frac{1}{p}} \\
& \leq r_{l}^{\frac{1}{q}}(q) . \sup _{t_{1} \in \mathbb{T}}\left[\int_{-\infty}^{0} \hat{e}_{\ominus\left(\frac{\bar{a}_{l} p}{2}\right)}(0, \sigma(z)) \times \frac{1}{K} \int_{t_{1}}^{t_{1}+K} \mid \sum_{m=1}^{n} b_{l m}(z+t) f_{m}\left(\psi_{m}(z+t)\right)\right. \\
& +\sum_{m=1}^{n} c_{l m}(z+t) g_{m}\left(\psi_{m}\left(z+t-\xi_{m}(z+t)\right)\right) \\
& \left.+\left.\sum_{m=1}^{n} d_{l m}(z+t) \int_{-\infty}^{z+t} N_{l m}(z+t-s) h_{m}\left(\psi_{m}(s)\right) \Delta s\right|_{\mathbb{H}} ^{p} \Delta(z+t) \Delta z\right]^{\frac{1}{p}} \\
& \leq r_{l}^{\frac{1}{q}}(q) \cdot \sup _{t_{2} \in \mathbb{T}}\left[\int_{-\infty}^{0} \hat{e}_{\ominus\left(\frac{\bar{a}_{l} p}{2}\right)}(0, \sigma(z)) \times \frac{1}{K} \int_{t_{2}}^{t_{2}+K} \mid \sum_{m=1}^{n} b_{l m}(\hat{t}) f_{m}\left(\psi_{m}(\hat{t})\right)\right. \\
& +\sum_{m=1}^{n} c_{l m}(\hat{t}) g_{m}\left(\psi_{m}\left(\hat{t}-\xi_{m}(\hat{t})\right)\right) \\
& \left.+\left.\sum_{m=1}^{n} d_{l m}(\hat{t}) \int_{-\infty}^{\hat{t}} N_{l m}(\hat{t}-s) h_{m}\left(\psi_{m}(s)\right) \Delta s\right|_{\mathbb{H}} ^{p} \Delta \hat{t} \Delta z\right]^{\frac{1}{p}} .
\end{aligned}
$$




$$
\begin{aligned}
& \leq r_{l}^{\frac{1}{q}}(q) \cdot r_{l}^{\frac{1}{p}}(p) \times\left(\sup _{t_{2} \in \mathbb{T}}\left[\frac{1}{K} \int_{t_{2}}^{t_{2}+K} \sum_{m=1}^{n}\left|b_{l m}(\hat{t})\right|_{\mathbb{H}}^{p}\left|f_{m}\left(\psi_{m}(\hat{t})\right)\right|_{\mathbb{H}}^{p} \Delta \hat{t}\right]^{\frac{1}{p}}\right. \\
& +\sup _{t_{2} \in \mathbb{T}}\left[\frac{1}{K} \int_{t_{2}}^{t_{2}+K} \sum_{m=1}^{n}\left|c_{l m}(\hat{t})\right|_{\mathbb{H}}^{p}\left|g_{m}\left(\psi_{m}\left(\hat{t}-\xi_{m}(\hat{t})\right)\right)\right|_{\mathbb{H}}^{p} \Delta \hat{t}\right]^{\frac{1}{p}} \\
& \left.+\sup _{t_{2} \in \mathbb{T}}\left[\frac{1}{K} \int_{t_{2}}^{t_{2}+K} \sum_{m=1}^{n}\left|d_{l m}(\hat{t})\right|_{\mathbb{H}}^{p} \int_{-\infty}^{\hat{t}}\left|N_{l m}(\hat{t}-s) h_{m}\left(\psi_{m}(s)\right) \Delta s\right|_{\mathbb{H}}^{p} \Delta \hat{t}\right]^{\frac{1}{p}}\right), \\
& \leq \max _{1 \leq l \leq n}\left\{r_{l}^{\frac{1}{q}}(q) \cdot r_{l}^{\frac{1}{p}}(p)\left[\sum_{m=1}^{n} b_{l m}^{*} L_{m}^{f}+\sum_{m=1}^{n} \frac{c_{l m}^{*} L_{m}^{g}}{\left(1-\xi_{m}^{*}\right)^{\frac{1}{p}}}+\sum_{m=1}^{n} d_{l m}^{*} L_{m}^{h} N^{*}\right]\right\} \times\|\|_{S^{p}}, \\
& \leq \varpi .\|\psi\|_{S^{p}} .
\end{aligned}
$$

On the other hand,

$$
\begin{aligned}
& \left\|\psi_{0}(t)\right\|_{S^{p}}=\sup _{t_{1} \in \mathbb{T}}\left(\frac{1}{K} \int_{t_{1}}^{t_{1}+K}\left|\int_{-\infty}^{t} \hat{e}_{\ominus a_{l}}(t, \sigma(z)) I_{l}(z)\right|_{\mathbb{H}}^{p} \Delta z \Delta t\right)^{\frac{1}{p}}, \\
& \leq r_{l}^{\frac{1}{q}}(q) \sup _{t_{1} \in \mathbb{T}}\left(\int_{-\infty}^{0} \hat{e}_{\ominus\left(\frac{\bar{a}_{l} p}{2}\right)}(0, \sigma(z)) \frac{1}{K} \int_{t_{1}}^{t_{1}+K}\left|I_{l}(z+t)\right|_{\mathbb{H}}^{p} \Delta t \Delta z\right)^{\frac{1}{p}}, \\
& \leq r_{l}^{\frac{1}{q}}(q) \sup _{t_{2} \in \mathbb{T}}\left(\int_{-\infty}^{0} \hat{e}_{\ominus\left(\frac{\bar{l}_{l} p}{2}\right)}(0, \sigma(z)) \frac{1}{K} \int_{t_{2}}^{t_{2}+K}\left|I_{l}(\bar{t})\right|_{\mathbb{H}}^{p} \Delta \bar{t} \Delta z\right)^{\frac{1}{p}}, \\
& \leq r_{l}^{\frac{1}{q}}(q) \cdot r_{l}^{\frac{1}{p}}(p) .\|I\|_{S^{p}<\rho_{I} . \quad \text { (Hölder's inequality) }}
\end{aligned}
$$

So, for any $\psi \in \mathbb{B}$, we have

$$
\|\psi\|_{S^{p}} \leq\left\|\psi-\psi_{0}\right\|_{S^{p}}+\left\|\psi_{0}\right\|_{S^{p}} \leq \frac{\varpi \rho_{I}}{1-\varpi}+\rho_{I}=\frac{\rho_{I}}{1-\varpi} .
$$

Hence,

$$
\left\|\left(\Pi_{\psi}\right)_{l}(t)-\psi_{0}(t)\right\|_{S^{p}} \leq \frac{\varpi \rho_{I}}{1-\varpi}
$$


which implies that $\left(\Pi_{\psi}\right)_{l} \in \mathbb{B}$.

Second step : we shall prove that $\left(\Pi_{\psi}\right)_{l}$ is a contraction mapping. In fact, For $\psi, \phi \in \mathbb{B}$, we get

$$
\begin{aligned}
& \left\|\left(\Pi_{\psi}\right)_{l}(t)-\left(\Pi_{\phi}\right)_{l}(t)\right\|_{S^{p}}= \\
& \sup _{t_{1} \in \mathbb{T}}\left[\frac{1}{K} \int_{t_{1}}^{t_{1}+K} \mid \int_{-\infty}^{t} \hat{e}_{\ominus a_{l}}(t, \sigma(z))\left(\sum _ { m = 1 } ^ { n } b _ { l m } ( z ) \left(f_{m}\left(\psi_{m}(z)\right)\right.\right.\right. \\
& \left.-f_{m}\left(\phi_{m}(z)\right)\right)+\sum_{m=1}^{n} c_{l m}(z)\left(g_{m}\left(\psi_{m}\left(z-\xi_{m}(z)\right)\right)-g_{m}\left(\phi_{m}\left(z-\xi_{m}(z)\right)\right)\right) \\
& \left.\left.+\sum_{m=1}^{n} d_{l m}(z) \int_{-\infty}^{z} N_{l m}(z-s)\left(h_{m}\left(\psi_{m}(s)\right)-h_{m}\left(\phi_{m}(s)\right)\right) \Delta s\right)\left.\Delta z\right|_{\mathbb{H}} ^{p} \Delta t\right]^{\frac{1}{p}} \\
& \leq \max _{1 \leq l \leq n}\left\{r_{l}^{\frac{1}{q}}(q) r_{l}^{\frac{1}{p}}(p)\left[\sum_{m=1}^{n} b_{l m}^{*} L_{m}^{f}+\sum_{m=1}^{n} \frac{c_{l m}^{*} L_{m}^{g}}{\left(1-\xi_{m}^{*}\right)^{\frac{1}{p}}}+\sum_{m=1}^{n} d_{l m}^{*} L_{m}^{h} N^{*}\right]\right\} \\
& \times\|\psi-\phi\|_{S^{p}} \leq \varpi .\|\psi-\phi\|_{S^{p}<1 .} .
\end{aligned}
$$

Hence, we obtain that $\left(\Pi_{\psi}\right)_{l}$ is a contraction mapping. Then, system (2.1) has a unique $S^{p}$-pseudo almost automorphic solution in the region $\mathbb{B}$. The proof is complete.

\subsection{Global $S^{p}$-exponential stability.}

Lemma 3.4 ( [31]). For any $u, v \in \mathbb{H}$, if $P \in \mathbb{H}^{n \times n}$ is a positive-definite Hermitian matrix, then

$$
\bar{u} v+\bar{v} u \leq \bar{u} P u+\bar{v} P^{-1} v
$$

Definition 3.1. The dynamical networks (2.1) is said to be $S^{p}$-globally exponentially stable, if there exist positive constants $\lambda$ with $\ominus \lambda \in \mathfrak{R}^{+}$and $M>0$ such that

$$
\|v(t)-u(t)\|_{S^{p}} \leq M \hat{e}_{\ominus \lambda}(t, 0), \quad \forall t \in(0, \infty)_{\mathbb{T}}
$$

Where $u()=.\left(u_{1}(),. u_{2}(),. \ldots, u_{n}().\right)$ is a Stepanov-like pseudo almost automorphic solution of QVFRNNs (2.1) on $\mathbb{T}$ and $v()=.\left(v_{1}(),. v_{2}(),. \ldots, v_{n}().\right)$ is an arbitrary solution of QVRNNs (2.1) on $\mathbb{T}$.

Theorem 3.3. Suppose that assumptions $\left(S_{1}\right)-\left(S_{5}\right)$ hold, and $\varpi<1$. Then the unique Stepanov-like pseudo almost automorphic solution of system (2.1) is $S^{p}$-globally exponentially stable on $\mathbb{T}$ whenever

$$
\begin{aligned}
\left(S_{6}\right) \frac{1}{a_{l}^{*}}-\check{a}_{l}< & -\sum_{m=1}^{n} a_{l}^{*}\left(b_{l m}^{*} L_{l}^{f}\right)^{2}-\sum_{m=1}^{n} \frac{a_{l}^{*}\left(c_{l m}^{*} L_{l}^{g}\right)^{2}}{1-\xi^{*}} \\
& -\sum_{m=1}^{n} a_{l}^{*}\left(d_{l m}^{*} L_{l}^{h}\right)^{2} \int_{0}^{\infty} N_{l m}^{2}(z) \Delta z .
\end{aligned}
$$


Proof. Let $u($.$) be the S^{p}$-pseudo almost automorphic solution on $\mathbb{T}$ and let $y($.$) be an arbitrary so-$ lution of sys.(2.1), $X()=.u()-.v(),. F(X())=.f(u())-.f(v()),. G(X())=.g(u())-.g(v()$.$) , and$ $H(X())=.h(u())-.h(v()$.$) . Let x \in[0, \infty)$, we define the function $x \mapsto D_{l}(x)$ as follows:

$$
\begin{aligned}
D_{l}(x) & =x+\frac{1}{a_{l}^{*}}-\check{a}_{l}+\sum_{m=1}^{n} a_{l}^{*}\left(b_{l m}^{*} L_{l}^{f}\right)^{2}+\frac{\exp (x(\bar{\mu}+\bar{\xi}))}{1-\xi^{*}} \sum_{m=1}^{n} a_{l}^{*}\left(c_{l m}^{*} L_{l}^{g}\right)^{2} \\
& +\sum_{m=1}^{n} a_{l}^{*}\left(d_{l m}^{*} L_{l}^{h}\right)^{2} \int_{0}^{\infty} N_{l m}^{2}(z) \hat{e}_{x}(\sigma(z), 0) \Delta z<0 .
\end{aligned}
$$

By $\left(S_{6}\right)$, we have $D_{l}(0)<0$. Since the function $D_{l}($.$) is continuous on [0, \infty)$, there exist $0<\lambda<$ $\min _{1 \leq l \leq n} \bar{a}_{l}$, such that $\Theta_{l}(\lambda)<0$.

Now, consider the Lyapunov function as follows :

$$
\begin{aligned}
V(t) & =\sum_{l=1}^{n}\left|X_{l}(t)\right|_{\mathbb{H}}^{2} \hat{e}_{\lambda}(t, 0) \\
& +\frac{\exp (\lambda(\bar{\mu}+\bar{\xi}))}{1-\xi^{*}} \sum_{l=1}^{n} \sum_{m=1}^{n} a_{l}^{*}\left(c_{l m}^{*} L_{l}^{g}\right)^{2} \int_{t-\xi(t)}^{t}\left|X_{l}(z)\right|_{\mathbb{H}}^{2} \hat{e}_{\lambda}(\sigma(z), 0) \Delta z \\
& +\sum_{l=1}^{n} \sum_{m=1}^{n}\left(d_{l m}^{*} L_{l}^{h}\right)^{2} \int_{0}^{\infty} N_{l m}^{2}(z) \int_{t-z}^{t}\left|X_{l}(s)\right|_{\mathbb{H}}^{2} \hat{e}_{\lambda}(\sigma(z), 0) \hat{e}_{\lambda}(\sigma(s), 0) \Delta s \Delta z
\end{aligned}
$$

\section{Consequently,}

$$
\begin{aligned}
& V^{\Delta}(t)=\lambda \hat{e}_{\lambda}(t, 0) \sum_{l=1}^{n}\left|X_{l}(t)\right|_{\mathbb{H}}^{2}+\hat{e}_{\lambda}(t, 0) \sum_{l=1}^{n} X_{l}^{\Delta}(t) \overline{X_{l}(t)} \\
& +\frac{\exp (\lambda(\bar{\mu}+\bar{\xi}))}{1-\xi^{*}} \sum_{l=1}^{n} \sum_{m=1}^{n} a_{l}^{*}\left(c_{l m}^{*} L_{l}^{g}\right)^{2} \\
& \times\left[\hat{e}_{\lambda}(t, 0)\left|X_{l}(t)\right|_{\mathbb{H}}^{2}-\left(1-\xi_{m}^{\Delta}(t)\right) \exp \left(-\lambda \xi_{m}(t)\right) \hat{e}_{\lambda}(t, 0)\left|X_{l}\left(t-\xi_{m}^{\Delta}(t)\right)\right|_{\mathbb{H}}^{2}\right] \\
& +\sum_{l=1}^{n} \sum_{m=1}^{n} a_{l}^{*}\left(d_{l m}^{*} L_{l}^{h}\right)^{2} \int_{0}^{\infty} N_{l m}^{2}(z)\left[\hat{e}_{\lambda}(t, 0) \hat{e}_{\lambda}(\sigma(z), 0)\left|X_{l}(t)\right|_{\mathbb{H}}^{2}-\hat{e}_{\lambda}(t, 0)\left|X_{l}(t-z)\right|_{\mathbb{H}}^{2}\right] \Delta z
\end{aligned}
$$

According to lemma (3.4) and by using $\left(S_{1}\right), \exp (\lambda \bar{\xi}) \cdot \exp \left(-\lambda \xi_{m}(t)\right)>1$, we have 


$$
\begin{aligned}
& V^{\Delta}(t) \leq \lambda \hat{e}_{\lambda}(t, 0) \sum_{l=1}^{n}\left|X_{l}(t)\right|_{\mathbb{H}}^{2}-\hat{e}_{\lambda}(t, 0) \sum_{l=1}^{n} \check{a}_{l}\left|X_{l}(t)\right|_{\mathbb{H}}^{2} \\
& +\hat{e}_{\lambda}(t, 0) \sum_{l=1}^{n}\left(\sum_{m=1}^{n} b_{l m}(t) F_{m}\left(X_{m}(t)\right) \cdot \overline{b_{l m}(t) F_{m}\left(X_{m}(t)\right)} \cdot a_{l}^{*}+\frac{X_{l}(t) \overline{X_{l}(t)}}{a_{l}^{*}}\right) \\
& +\hat{e}_{\lambda}(t, 0) \sum_{l=1}^{n}\left(\sum_{m=1}^{n} \overline{c_{l m}(t) G_{m}\left(X_{m}\left(t-\xi_{m}(t)\right)\right)} \cdot c_{l m}(t) G_{m}\left(X_{m}\left(t-\xi_{m}(t)\right)\right) \cdot a_{l}^{*}\right. \\
& \left.+\frac{X_{l}(t) \overline{X_{l}(t)}}{a_{l}^{*}}\right)+\hat{e}_{\lambda}(t, 0) \sum_{l=1}^{n}\left(\sum_{m=1}^{n} \overline{d_{l m}(t) \int_{-\infty}^{t} N_{l m}(t-z) H_{m}\left(X_{m}(t)\right) \Delta z \cdot d_{l m}(t)}\right. \\
& \left.\times \int_{-\infty}^{t} N_{l m}(t-z) H_{m}\left(X_{m}(t)\right) \Delta z \cdot a_{l}^{*}+\frac{X_{l}(t) \overline{X_{l}(t)}}{a_{l}^{*}}\right)+\frac{\exp (\lambda \bar{\mu})}{1-\xi^{*}} \sum_{l=1}^{n} \sum_{m=1}^{n} a_{l}^{*}\left(c_{l m}^{*} L_{l}^{g}\right)^{2} \\
& \times\left(\exp (\lambda \bar{\xi}) \hat{e}_{\lambda}(t, 0)\left|X_{l}(t)\right|_{\mathbb{H}}^{2}-\left(1-\xi_{m}^{\Delta}(t)\right) \hat{e}_{\lambda}(t, 0)\left|X_{l}\left(t-\xi_{m}^{\Delta}(t)\right)\right|_{\mathbb{H}}^{2}\right) \\
& +\sum_{l=1}^{n} \sum_{m=1}^{n} a_{l}^{*}\left(d_{l m}^{*} L_{l}^{h}\right)^{2} \int_{0}^{\infty} N_{l m}^{2}(z)\left(\hat{e}_{\lambda}(t, 0) \hat{e}_{\lambda}(\sigma(z), 0)\left|X_{l}(t)\right|_{\mathbb{H}}^{2}-\hat{e}_{\lambda}(t, 0)\left|X_{l}(t-z)\right|_{\mathbb{H}}^{2}\right) \Delta z, \\
& \leq \hat{e}_{\lambda}(t, 0) \sum_{l=1}^{n}\left(\lambda+\frac{1}{a_{l}^{*}}-\check{a}_{l}+\sum_{m=1}^{n} a_{l}^{*}\left(b_{l m}^{*} L_{l}^{f}\right)^{2}+\frac{\exp (\lambda(\bar{\mu}+\bar{\xi}))}{1-\xi^{*}} \sum_{m=1}^{n} a_{l}^{*}\left(c_{l m}^{*} L_{l}^{g}\right)^{2}\right. \\
& \left.+\sum_{m=1}^{n} a_{l}^{*}\left(d_{l m}^{*} L_{l}^{h}\right)^{2} \int_{0}^{\infty} N_{l m}^{2}(z) \hat{e}_{\lambda}(\sigma(z), 0) \Delta z\right)\left|X_{l}(t)\right|_{\mathbb{H}}^{2}, \\
& <0 .
\end{aligned}
$$

For this reason, $\sum_{l=1}^{n}\left|X_{l}(t)\right|_{\mathbb{H}}^{2} \leq \hat{e}_{\ominus \lambda}(t, 0) V(0), \quad$ with $\ominus \lambda \in \mathfrak{R}^{+}, l=1, \ldots, n$.

It follows that $\sum_{l=1}^{n}\left|X_{l}(t)\right|_{\mathbb{H}}^{p} \leq \hat{e}_{\ominus p \lambda}(t, 0) V(0)^{p}, p \geq 2$. Thus,

$$
\begin{aligned}
\sum_{l=1}^{n} \frac{1}{K} \int_{t-\bar{\xi}}^{t-\bar{\xi}+K}\left|X_{l}(z)\right|_{\mathbb{H}}^{p} \Delta z & \leq \sum_{l=1}^{n} \frac{1}{K} \int_{t}^{t+K}\left|X_{l}(z)\right|_{\mathbb{H}}^{p} \Delta z \\
& \leq \int_{t}^{t+K} \frac{\hat{e}_{\ominus p \lambda}(t, 0) V(0)^{p}}{K}
\end{aligned}
$$

Furthermore,

$$
\sum_{l=1}^{n} \frac{1}{K} \int_{t}^{t+K}\left|X_{l}(z)\right|_{\mathbb{H}}^{p} \Delta z \leq \frac{V(0)^{p} \hat{e}_{\ominus \lambda p}(t, 0)}{K} \frac{(\exp (-\lambda p K)-1)}{\ominus \lambda p}
$$

Besides,

$$
\max _{l=1, \ldots, n} \sup _{t_{1} \in \mathbb{T}}\left(\frac{1}{K} \int_{t_{1}}^{t_{1}+K}\left|X_{l}(z)\right|_{\mathbb{H}}^{p} \Delta z\right)^{\frac{1}{p}} \leq \frac{V(0) \hat{e}_{\ominus \lambda}(t, 0)}{K^{\frac{1}{p}}}\left(\frac{(\exp (-\lambda p K)-1)}{\ominus \lambda p}\right)^{\frac{1}{p}}
$$


We claim that

$$
\|X\|_{S^{p}} \leq \frac{V(0) \hat{e}_{\ominus \lambda}(t, 0)}{K^{\frac{1}{p}}}\left(\frac{(\exp (-\lambda p K)-1)}{\ominus \lambda p}\right)^{\frac{1}{p}} \leq M \hat{e}_{\ominus \lambda}(t, 0) .
$$

Where $M=\frac{V(0)}{K^{\frac{1}{p}}}\left(\frac{(\exp (-\lambda p K)-1)}{\ominus \lambda p}\right)^{\frac{1}{p}}$. Therefore, the unique Stepanov-like pseudo almost automorphic solution of QVRNNs (2.1) is $S^{p}$-globally exponentially stable on time scales. This completes the proof.

Remark 3.1. To our knowledge, there have been no results concentrated on the Stepanov-like almost pariodic, Stepanov-like pseudo almost periodic, and weighted Stepanov-like pseudo-almost periodic solution for QVFRNNs with Mixed Delays on time scales. As a consequence, the obtained results in this work are essentially new and the methods used in this paper can also be applied to study the Stepanov-like pseudo almost automorphic solution on time scales for some other models of dynamical NNs.

Corollary 3.1. If $\mathbb{H}=\mathbb{R}$, then sys (2.1) is a real-valued RNNs on $\mathbb{T}$. Assume that the following conditions hold

$\left(F_{1}\right):$ For all $1 \leq l \leq n$, the function $\xi_{m}(.) \in S^{p} A A(\mathbb{T}, \mathbb{R}) \cap C_{r d}^{1}(\mathbb{T}, \mathbb{R})$, such that

$$
0 \leq \xi_{m}(.) \leq \bar{\xi}, \quad 0 \leq \xi^{*}-\xi_{m}^{\Delta}(.)<1-\xi_{m}^{\Delta}(.)
$$

$\left(F_{2}\right):$ For all $1 \leq l, m \leq n$, the functions $a_{l m}(),. b_{l m}(),. c_{l m}(),. d_{l m}(.) \in S^{p} P A A(\mathbb{T}, \mathbb{R})$.

$\left(F_{3}\right)$ : There exist positive constants $L_{l}^{f}, L_{l}^{g}, L_{h_{l}}$ such that for any $u, v \in \mathbb{R}$, the activity functions $f_{l}$, $g_{l}, h_{l} \in C_{r d}(\mathbb{R}, \mathbb{R})$ satisfying

$$
\begin{aligned}
& \left|f_{l}(u)-f_{l}(v)\right| \leq L_{l}^{f}|u-v|, \\
& \left|g_{l}(u)-g_{l}(v)\right| \leq L_{l}^{g}|u-v|, \\
& \left|h_{l}(u)-h_{l}(v)\right| \leq L_{l}^{h}|u-v| .
\end{aligned}
$$

Furthermore, we suppose that $f_{l}(0)=g_{l}(0)=h_{l}(0)=0$.

$\left(F_{4}\right):$ For all $1 \leq l, m \leq n$, the delay kernel $N_{l m}:[0,+\infty)_{\mathbb{T}} \rightarrow[0,+\infty)$ is rd-continuous, $\Delta-$ integrable and there exists $\lambda>0$ such that $\int_{0}^{+\infty} N_{l m}(z) \Delta z=N^{*}$. 
$\left(F_{5}\right): \varpi<1$, and

$$
\begin{aligned}
\frac{1}{a_{l}^{*}}-\check{a}_{l} & <-\sum_{m=1}^{n} a_{l}^{*}\left(b_{l m}^{*} L_{l}^{f}\right)^{2}-\sum_{m=1}^{n} \frac{a_{l}^{*}\left(c_{l m}^{*} L_{l}^{g}\right)^{2}}{1-\xi^{*}} \\
& -\sum_{m=1}^{n} a_{l}^{*}\left(d_{l m}^{*} L_{l}^{h}\right)^{2} \int_{0}^{\infty} N_{l m}^{2}(z) \Delta z .
\end{aligned}
$$

Then, sys.(2.1) has a unique $S^{p}$-pseudo almost automorphic solution on time scales and this solution is $S^{p}$-globally exponentially stable.

Corollary 3.2. If $\mathbb{H}=\mathbb{C}$, then sys (2.1) is a complex-valued RNNs on $\mathbb{T}$. Assume that the following conditions hold

$\left(A_{1}\right):$ For all $1 \leq l \leq n$, the function $\xi_{m}(.) \in S^{p} A A(\mathbb{T}, \mathbb{C}) \cap C_{r d}^{1}(\mathbb{T}, \mathbb{C})$, such that

$$
0 \leq \xi_{m}(.) \leq \bar{\xi}, \quad 0 \leq \xi^{*}-\xi_{m}^{\Delta}(.)<1-\xi_{m}^{\Delta}(.) .
$$

$\left(A_{2}\right)$ : For all $1 \leq l, m \leq n$, the functions $a_{l m}(),. b_{l m}(),. c_{l m}(),. d_{l m}(.) \in S^{p} P A A(\mathbb{T}, \mathbb{C})$.

$\left(A_{3}\right)$ : There exist positive constants $L_{l}^{f}, L_{l}^{g}, L_{h_{l}}$ such that for any $u, v \in \mathbb{C}$, the activity functions $f_{l}$, $g_{l}, h_{l} \in C_{r d}(\mathbb{C}, \mathbb{C})$ satisfying

$$
\begin{aligned}
& \left|f_{l}(u)-f_{l}(v)\right|_{\mathbb{C}} \leq L_{l}^{f}|u-v|_{\mathbb{C}}, \\
& \left|g_{l}(u)-g_{l}(v)\right|_{\mathbb{C}} \leq L_{l}^{g}|u-v|_{\mathbb{C}}, \\
& \left|h_{l}(u)-h_{l}(v)\right|_{\mathbb{C}} \leq L_{l}^{h}|u-v|_{\mathbb{C}} .
\end{aligned}
$$

And $f_{l}(0)=g_{l}(0)=h_{l}(0)=0$.

$\left(A_{4}\right):$ For all $1 \leq l, m \leq n$, the delay kernel $N_{l m}:[0,+\infty)_{\mathbb{T}} \rightarrow[0,+\infty)$ is rd-continuous, $\Delta$-integrable and there exists $\lambda>0$ such that $\int_{0}^{+\infty} N_{\operatorname{lm}}(z) \Delta z=N^{*}$.

$\left(A_{5}\right): \varpi<1$, and

$$
\begin{aligned}
1 a_{l}^{*}-\check{a}_{l} & <-\sum_{m=1}^{n} a_{l}^{*}\left(b_{l m}^{*} L_{l}^{f}\right)^{2}-\sum_{m=1}^{n} \frac{a_{l}^{*}\left(c_{l m}^{*} L_{l}^{g}\right)^{2}}{1-\xi^{*}} \\
& -\sum_{m=1}^{n} a_{l}^{*}\left(d_{l m}^{*} L_{l}^{h}\right)^{2} \int_{0}^{\infty} N_{l m}^{2}(z) \Delta z .
\end{aligned}
$$

Where

$$
b_{l m}^{*}=\sup _{t \in \mathbb{T}}\left|b_{l m}(t)\right|_{\mathbb{C}}, \quad c_{l m}^{*}=\sup _{t \in \mathbb{T}}\left|c_{l m}(t)\right|_{\mathbb{C}}, \quad d_{l m}^{*}=\sup _{t \in \mathbb{T}}\left|d_{l m}(t)\right|_{\mathbb{C}}
$$

Therefore, sys.(2.1) has a unique $S^{p}$-pseudo almost automorphic solution on time scales and this solution is $S^{p}$-globally exponentially stable. 


\section{Numerical ExAMPle}

In this section, we give two examples to illustrate the feasibility and effectiveness of our results derived in the previous sections.

Example 4.1. Let $n=l=m=1, p=q=2, \alpha=1$ and the coefficients are taken as follows:

$$
\begin{aligned}
& f_{1}\left(x_{1}\right)=g_{1}\left(x_{1}\right)=\frac{1}{8} \sin ^{2}(t), h_{1}\left(x_{1}\right)=\frac{1}{8}(t-1) j, \xi_{1}=1, a_{1}=1+j+i+k, \\
& b_{11}(t)=\exp (-t)+i \cos (t), c_{11}(t)=j \sin (t), d_{11}(t)=i(\sin (t)+1), \\
& I_{11}=\exp (-t) \cos (t)+i \sin (t)+j \exp (-t) \cos (t)+2 k \cos (t), N_{l m}=\exp (-t) .
\end{aligned}
$$

If $\mathbb{T}=\mathbb{R}:$ We have $\mu(t)=\sigma(t)-t=t-t=0 \quad \forall t \in \mathbb{T}$, then $\bar{\mu}=0$

we have

$$
\begin{aligned}
& L_{1}^{f}=L_{1}^{g}=L_{1}^{h}=\frac{1}{8}, \quad \xi_{1}^{*}=0, \quad N^{*}=1, \\
& b_{11}^{+}=d_{11}^{+}=1 \quad c_{11}^{+}=2, \quad \bar{a}_{1}=a_{1}^{*}=4 \quad \check{a}_{1}=8 .
\end{aligned}
$$

Moroever, $L^{*} \simeq 0.156<1$. 


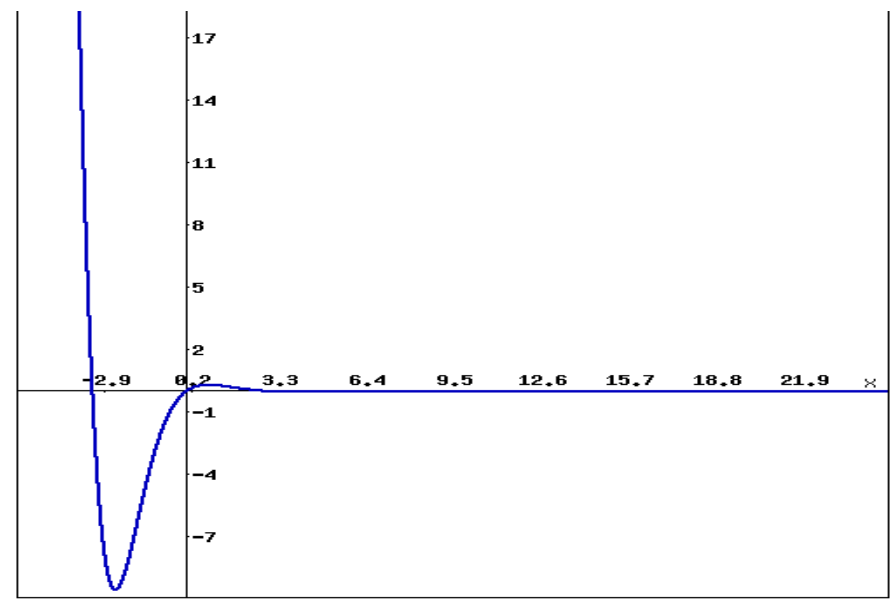

FIGURE 1. Behavior of the state variable $x_{1}^{R}$ of QVFRNNs (2.1)

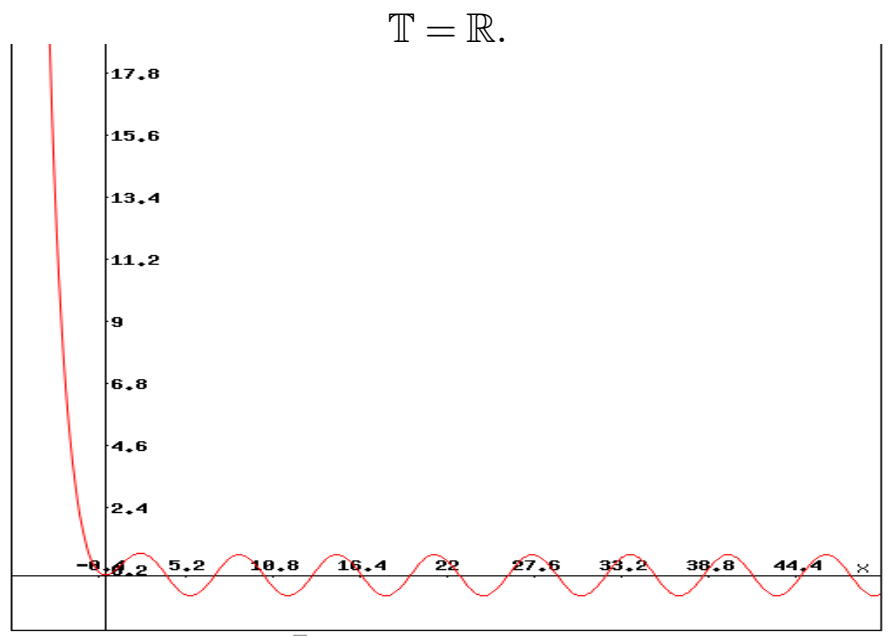

FIGURE 2. Behavior of the state variable $x_{1}^{I}$ of QVFRNNs (2.1)

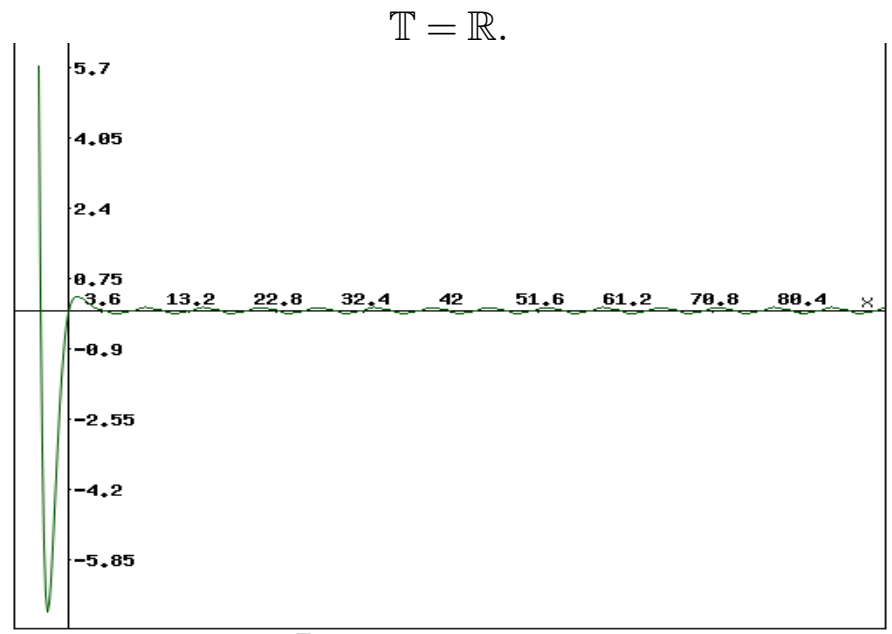

FIGURE 3. Behavior of the state variable $x_{1}^{J}$ of QVFRNNs (2.1)

$$
\mathbb{T}=\mathbb{R} .
$$




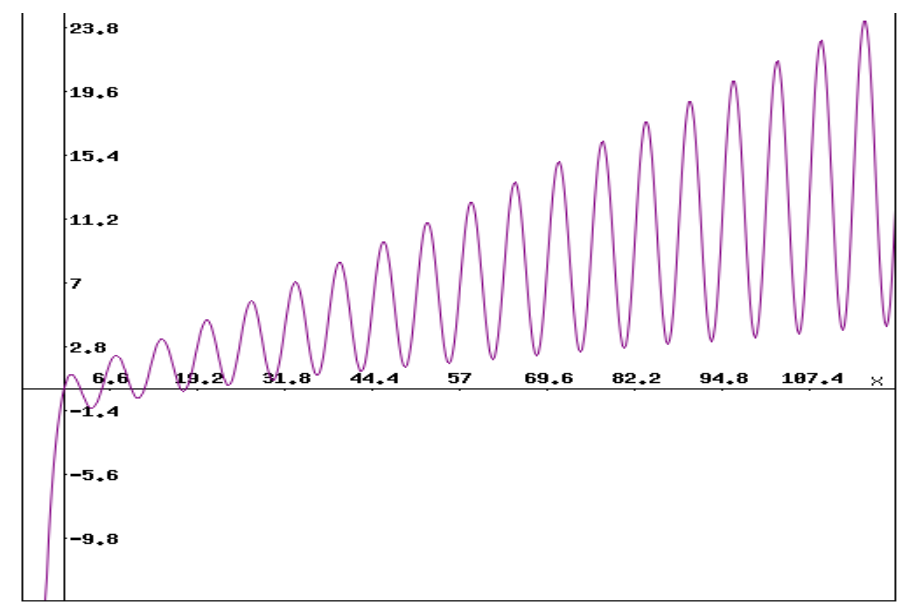

FIGURE 4. Behavior of the state variable $x_{1}^{K}$ of QVFRNNs (2.1)

$$
\mathbb{T}=\mathbb{R}
$$

If $\mathbb{T}=\mathbb{Z}:$ We have $\mu(t)=\sigma(t)-t=t+1-t=1 \quad \forall t \in \mathbb{T}$, then $\bar{\mu}=1$ Hence, $L^{*} \simeq 0.781<1$, and $\frac{1}{a_{l}^{*}}-\check{a}_{l} \simeq-7.75<-0.43$. Then, whether $\mathbb{T}=\mathbb{R}$ or $\mathbb{T}=\mathbb{Z}$, all the conditions of Theorems (3.2) and (3.3) are satisfied. Thus, we know that system (2.1) has a unique Stepanov-like pseudo almost automorphic solution, which is $S^{p}$ globally exponentially stable. So, the discrete-time neural network and its continuous-time analogue have the same dynamical behaviors for the $S^{p}$-pseudo almost automorphy.

Example 4.2. Let $n=2, l=1 ; 2, p=q=2, \lambda=1$ and the coefficients are taken as follows:

$$
\begin{aligned}
& f_{m}\left(x_{m}(.)\right)=g_{m}\left(x_{m}(.)\right)=h_{m}\left(x_{m}(.)\right) \\
& =\frac{1}{5}\left(\cos \left(x_{m}^{I}(.)\right)+j \tan \left(x_{m}^{J}(.)\right)+k \sin \left(x_{m}^{J}(.)-x_{m}^{R}(.)\right)\right), \\
& a_{1}(.)=10+3 i \sin (\sqrt{2} t), a_{2}(.)=6+k \cos (\pi t), \\
& I_{l}(t)=\frac{2+\exp (i t)+\exp (i \sqrt{2 t})}{|2+\exp (i t)+\exp (i \sqrt{2 t})|}+\exp (-t), \\
& N_{l m}=\exp (-s), b_{l m}=\frac{1}{20}\left[\begin{array}{cc}
\cos \left(\frac{1}{2+\sin (t)+\sin (\sqrt{2} t)}\right)+\exp (-t) & \frac{1}{1+t^{2}}+j \cos (\sqrt{2} t) \\
k \cos (\sqrt{3} t)+j \cos (\sqrt{5} t) & \sin (t)+\exp (-2 t)
\end{array}\right], \\
& c_{l m}=\frac{1}{20}\left[\begin{array}{cc}
j \cos (\sqrt{5} t)+\exp \left(-t \cos ^{2}(t)\right) & k \sin \left(\frac{1}{2+\sin (t)+\sin (\sqrt{5} t)}\right)+\exp \left(-t^{4}\right) \\
i \sin \left(\frac{1}{2+\sin (t)+\sin (\sqrt{5} t)}\right)+i \exp \left(-t^{4}\right) & \cos (\pi t)+k \exp \left(-t^{4}\right)
\end{array}\right]
\end{aligned}
$$




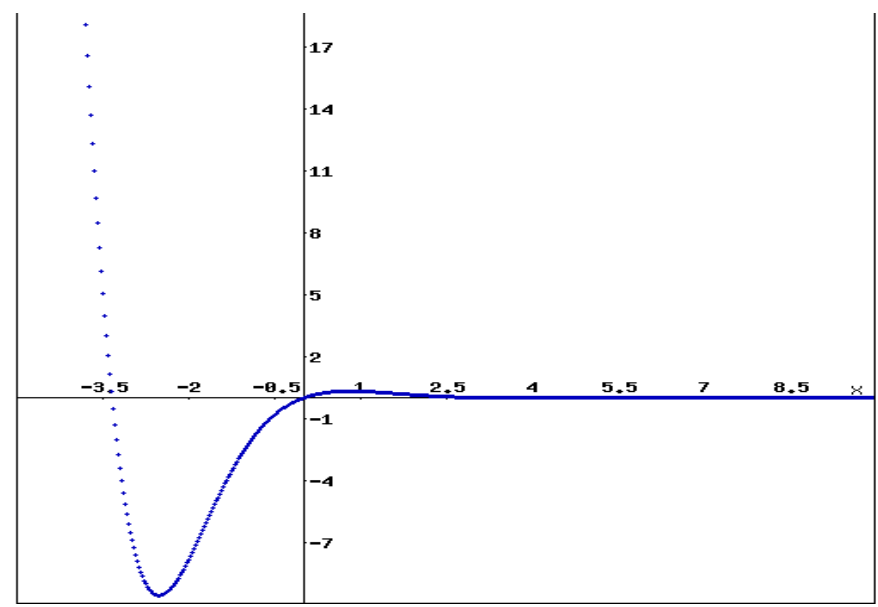

FIGURE 5. Behavior of the state variable $x_{1}^{R}$ of QVFRNNs (2.1)

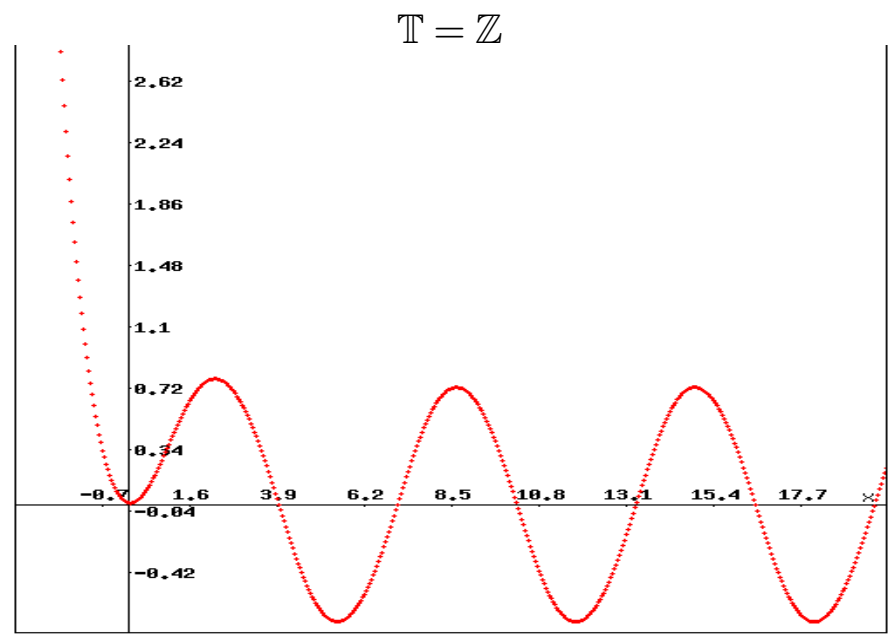

FIGURE 6. Behavior of the state variable $x_{1}^{I}$ of QVFRNNs (2.1)

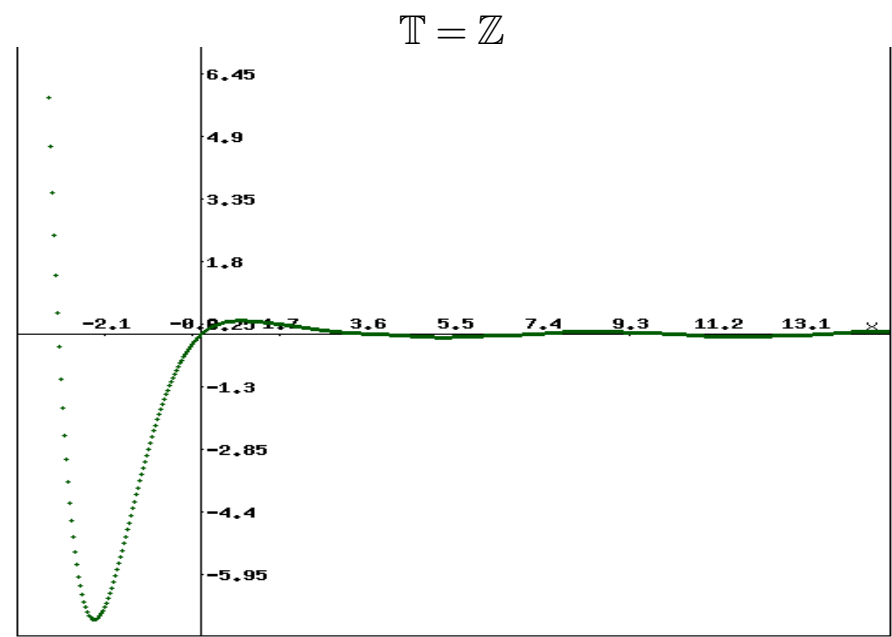

FIGURE 7. Behavior of the state variable $x_{1}^{J}$ of QVFRNNs (2.1)

$$
\mathbb{T}=\mathbb{Z}
$$




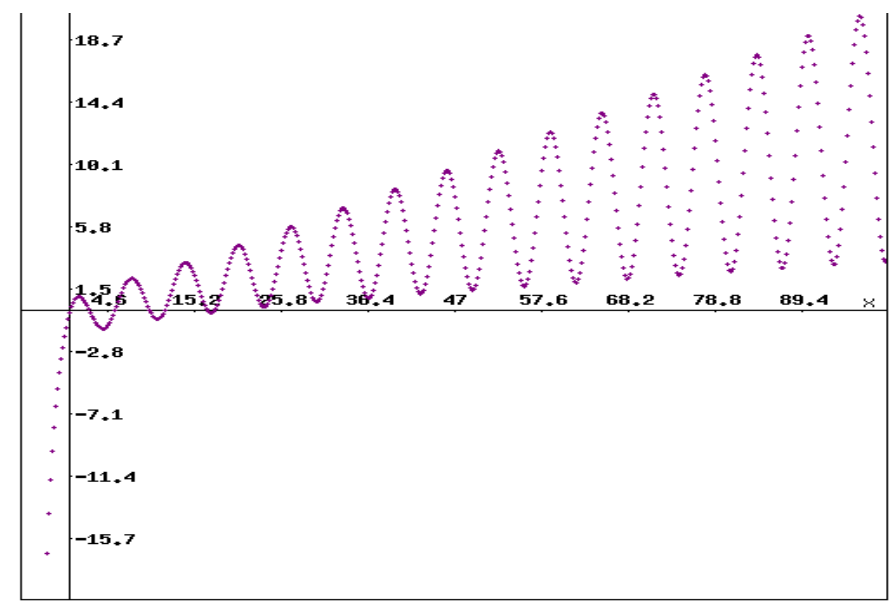

FIGURE 8. Behavior of the state variable $x_{1}^{K}$ of QVFRNNs (2.1)

$$
\begin{gathered}
\mathbb{T}=\mathbb{Z} \\
d_{l m}=\frac{1}{20}\left[\begin{array}{cc}
i \sin (\sqrt{6} t)+\exp \left(-t^{2} \sin ^{2}(t)\right) & \frac{1}{1+t^{2}}+j \sin (\sqrt{8} t) \\
j \sin (\sqrt{8} t)+k \cos (\pi t)+\exp \left(-t^{4} \cos ^{4}(t)\right) & \sin (t)+i \cos (t)+\exp \left(-t^{4} \cos ^{2}(t)\right)
\end{array}\right] .
\end{gathered}
$$

Then, we have

$$
\bar{a}_{1}=7, \bar{a}_{2}=5, \check{a}_{1}=12, \check{a}_{2}=10, a_{1}^{*}=13, a_{2}^{*}=7, L_{m}^{f}=L_{m}^{g}=L_{m}^{h}=\frac{1}{5}, N^{*}=1,
$$

$b_{l m}^{*}=c_{l m}^{*}=\frac{1}{20}\left[\begin{array}{ll}2 & 2 \\ 2 & 2\end{array}\right], d_{l m}^{*}=\frac{1}{20}\left[\begin{array}{ll}2 & 2 \\ 3 & 3\end{array}\right]$,

If $\mathbb{T}=\mathbb{R}:$ Take $\xi_{1}(t)=\xi_{2}(t)=1$, then $\xi_{1}^{*}=\xi_{2}^{*}=0$. Hence, $\varpi \simeq \max \{0.017 ; 0.028\}=0.017<1$.

If $\mathbb{T}=\mathbb{Z}:$ Take $\xi_{1}(t)=\cos ^{2}\left(\pi t+\frac{\pi}{2}\right), \xi_{2}(t)=1$, then $\xi_{1}^{*}=\xi_{1}^{*}=0$. Moreover, $\varpi \simeq \max \{0.137 ; 0.168\}=$ $0.168<1$.

Besides, $\frac{1}{a_{1}^{*}}-\check{a_{1}} \simeq-11.92<-0.036$ and $\frac{1}{a_{2}^{*}}-\check{a_{2}} \simeq-9.85<-0.013$.

Therefore, all of the conditions of theorem (3.2) and (3.3) are satisfied. So, system (2.1) has a unique $S^{p}$-pseudo almost automorphic solution which is $S^{p}$-globally exponentially stable.

\section{REFERENCES}

[1] A. Cabada, and D. Vivero, Expression of the Lebesgue D-Integral on Time Scales as a Usual Lebesgue Intregral, Application to the Calculus of D-Antiderivatives. Math. Comput. Model. 43 (2006), 194-207.

[2] L.O. Chua and L. Yang, Cellular neural networks: theory. IEEE Trans. Circuits Syst. 35(10) (1988), 1257-1272

[3] W.H. Chen, S. Luo, W.X. Zheng, Impulsive Synchronization of Reaction-Diffusion Neural Networks With Mixed Delays and Its Application to Image Encryption. IEEE Transactions on Neural Networks and Learning Systems, vol. 27 (2016), pp. 2696-2710.

[4] A. Deniz and U. Ufuktepe, Lebesgue-Stieltjes Measure on Time Scales, Turk. J. Math. 33 (2009), 27-40. 
[5] M. Adivar and Y.N. Raffoul, Existence results for periodic solution of integro-dynamic equations on time scales, Ann. Mat. 188 (2009), 543-559.

[6] M. Bohner, V.S. Hari Rao and S. Sanyal, Global Stability of Complex-Valued Neural Networks on Time Scales, Differ. Equ. Dyn. Syst. 19 (2011), 3-11.

[7] N. Bertschinger and T. Natschlager, Real-Time Computation at the Edge of Chaos in Recurrent Neural Networks, Neural Comput. 16 (2014), 1413-1436.

[8] T. Diagana and M. Zitane, Stepanov-like pseudo-almost automorphic functions in Lebesgue spaces with variable exponents $L^{p(x)}$, Electron. J. Diff. Equ. 2013 (2013), No. 188, 1-20.

[9] M. Es-saiydy, M. Zitane, Weighted Stepanov-Like Pseudo Almost Periodicity on Time Scales and Applications, Differ. Equ. Dyn. Syst. (2020). https://doi.org/10.1007/s12591-020-00543-7.

[10] M. Es-saiydy and M. Zitane, Existence and Global Exponential Stability of Unique Weighted Stepanov-Like Pseudo Almost Periodic Solution Of Quaternion-Valued for Fuzzy Recurrent Neural Networks with Mixed Time-Varying Delays on time scales, submitted.

[11] M. Es-saiydy and M. Zitane, A New Composition Theorem for Weighted Stepanov-like Pseudo Almost Periodic Functions on Time Scales and Applications, submitted.

[12] M. Es-saiydy and M. Zitane,Stepanov-like Almost Automorphy and Stepanov-like Pseudo Almost Automorphy on Time Scales with Applications to Some Abstract Dynamic Equations, submitted.

[13] K. Guan, Global power-rate synchronization of chaotic neural networks with proportional delay via impulsive control. Neurocomputing, 283 (2018), 256-265.

[14] A. Hirose, Complex-valued Neural Networks, Springer, 2012.

[15] C. Huang, Y. Qiao, L. Huang, and al. Dynamical behaviors of a food-chain model with stage structure and time delays. Adv. Differ. Equ. 2018 (2018), 186.

[16] S.G. Hoggar, Mathematics for Computer Graphics, Cambridge University Press, Cambridge, UK, 1992.

[17] S. Hilger, Analysis on measure chains-a unified approach to continuous and discrete calculus. Results Math. 18 (1990), 18-56.

[18] W.R. Hamilton, Lectures on Quaternions, in s and Smith, Hodges, Dublin, 1853.

[19] B. Jackson, Partial dynamic equations on time scales, J. Comput. Appl. Math. 186 (2006), 391-415.

[20] J. Liu, X. Liu, W.C. Xie, Global convergence of neural networks with mixed time-varying delays and discontinuous neuron activations. Inform. Sci. 183 (2012), 92-105.

[21] L. Luo, H. Feng, and L. Ding, Color image compression based on quaternion neural network principal component analysis, in Proceedings of the 2010 International Conference on Multimedia Technology, ICMT 2010, China, October 2010.

[22] T. Lindblad and J.M. Kinser, Image Processing Using Pulse-Coupled Neural Networks, Springer, 2005.

[23] Y. Li, X. Meng, Existence and Global Exponential Stability of Pseudo Almost Periodic Solutions for Neutral Type Quaternion-Valued Neural Networks with Delays in the Leakage Term on Time Scales, Complexity. 2017 (2017), 9878369.

[24] P.D. McNelis, Neural Networks in Finance: Gaining Predictive Edge in the Market, Academic Press, 2005. 
[25] R. Mukundan, Quaternions: From Classical Mechanics to Computer Graphics, and Beyond, in Proceedings of the 7th Asian Technology Conference in Mathematics, (2002), 95-105.

[26] X. Meng and Y. Li, Periodic solution for a Class of Singular Hamiltonian Systems on Time Scales, J. Math. 2014 (2014), Article ID 573517, 7 pages.

[27] J.R. Rabunal and J. Dorrado, Artificial Neural Networks in Real-life Applications, Idea Group Publishing, 2005.

[28] S.S.P. Rattan and W.W. Hsieh, Complex-valued neural networks for nonlinear complex principal component analysis, Neural Networks, 18 (2005), 61-69.

[29] S. Ruseti, M. Dascalu1, A.M. Johnson, R. Balyan, K.J. Kopp, D.S. McNamara, S.A. Crossley and S. Trausan-Matu1, Predicting Question Quality Using Recurrent Neural Networks. In C. Penstein Rosé, R. Martínez-Maldonado, H.U. Hoppe, R. Luckin, M. Mavrikis, K. Porayska-Pomsta,...B. du Boulay (Eds.), Artificial Intelligence in Education (Vol.10947, pp. 491-502). https://doi.org/10.1007/978-3-319-93843-1-36.

[30] A. Sudbery, Quaternionic analysis, Mathematical Proc. Camb. Phil. Soc. 85 (1979), no. 2, 199-224.

[31] Q. Song and X. Chen, Multistability analysis of quaternion-valued neural networks with time delays. IEEE Trans. Neural Netw. Learn Syst. 29(11) (2018), 5430-5440.

[32] S. Shen, B. Li and Y. Li, Anti-Periodic Dynamics of Quaternion-Valued Fuzzy Cellular Neural Networks with Time-Varying Delays on Time Scales, Discrete Dyn. Nat. Soc. 2018 (2018), Article ID 5290786, 14 pages.

[33] H. Toshani and M. Farrokhi, Real-time inverse kinematics of redundant manipulators using neural networks and quadratic programming: A Lyapunov-based approach, Robot. Auton. Syst. 62 (2014), 766-781.

[34] C. Xu,and $\mathrm{Y}$. $\mathrm{Wu}$, On almost automorphic solutions for cellular neural networks with time-varying delays in leakage terms on time scales. J. Intell. Fuzzy Syst. 30 (2016), 423-436.

[35] C. YIN, Y. ZHU, J. FEI, AND X. HE, A Deep Learning Approach for Intrusion Detection Using Recurrent Neural Networks, in IEEE Access, vol. 5 (2017), pp. 21954-21961.

[36] H. Zhu, Q. Zhu, X. Sun and Hongwei Zhou, Existence and exponential stability of pseudo almost automorphic solutions for Cohen-Grossberg neural networks with mixed delays. Adv. Differ. Equ. 2016 (2016), 120.

[37] M. Zitane, A generalization of weighted Stepanov-like pseudo-almost automorphic space, New Zealand J. Math. 48 (2018), 129-155. 